Bull. Soc. math. France

133 (1), 2005, p. 87-120

\title{
ENTROPY MAXIMISATION PROBLEM FOR QUANTUM RELATIVISTIC PARTICLES
}

\author{
By Miguel Escobedo, Stéphane Mischler \& Manuel A. Valle
}

\begin{abstract}
The entropy of an ideal gas, both in the case of classical and quantum particles, is maximised when the number particle density, linear momentum and energy are fixed. The dispersion law energy to momentum is chosen as linear or quadratic, corresponding to non-relativistic or relativistic behaviour.

RÉSUMÉ (Maximisation d'entropie pour particules relativistes quantiques)

L'entropie d'un gaz idéal de particules, classiques ou quantiques, est maximisée lorsque la densité du nombre de particules, l'impulsion et l'énergie sont fixées. La loi de dispersion qui relie l'impulsion et l'énergie est linéaire ou quadratique, selon que le comportement des particules est non relativiste ou relativiste.
\end{abstract}

Texte reçu le 9 novembre 2002, accepté le 10 janvier 2003

Miguel Escobedo, Departamento de Matemáticas, Universidad del País Vasco, Apartado 644 Bilbao 48080 (Spain) • E-mail : mtpesmam@lg.ehu.es Stéphane Mischler, Ceremade, Université Paris IX-Dauphine, place du Maréchal de Lattre de Tassigny, 75016 Paris(France) • E-mail : mischler@ceremade.dauphine.fr Manuel A. Valle, Departamento de Física Teórica e Historia de la Ciencia, Universidad del País Vasco, Apartado 644 Bilbao 48080 (Spain) • E-mail : wtpvabam@lg. ehu.es

2000 Mathematics Subject Classification. - 82B40, 82C40, 83-02.

Key words and phrases. - Entropy, maximisation problem, moments, bosons, fermions.

The authors were partially supported by CNRS and UPV through a PIC between the Universidad del País Vasco and the École Normale Supérieure. The work of M. Escobedo is supported by TMR contract HCL \# ERBFMRXCT960033, DGES grant PB96-0663 and UPV 127.310-EB035/99. 


\section{Introduction}

We are interested in the maximisation problem for the quantum or nonquantum entropy functional

$$
H(g):=\int_{\mathbb{R}^{3}} h(g(p)) \mathrm{d} p, \quad h(g)=\tau^{-1}(1+\tau g) \ln (1+\tau g)-g \ln g,
$$

where $\tau \in \mathbb{R}$, under the relativistic or non-relativistic moments constraint

$$
\left(\begin{array}{l}
N(g) \\
P(g) \\
E(g)
\end{array}\right):=\int_{\mathbb{R}^{3}}\left(\begin{array}{c}
1 \\
p \\
\mathcal{E}(p)
\end{array}\right) g(p) \mathrm{d} p=\left(\begin{array}{c}
N \\
P \\
E
\end{array}\right),
$$

where $N>0$ is the total number (or mass) of particles, $P \in \mathbb{R}^{3}$ is the mean momentum and $E>0$ is the total energy. Depending of whether particles are considered to be relativistic or not the energy $\mathcal{E}(p)$ of a particle having momentum $p \in \mathbb{R}^{3}$ is defined by

$$
\mathcal{E}(p)=\mathcal{E}_{n r}(p)=\frac{|p|^{2}}{2 m},
$$

for a non-relativistic particle, and by

$$
\mathcal{E}(p)=\mathcal{E}_{r}(p)=\gamma m c^{2}, \quad \gamma=\sqrt{1+\frac{|p|^{2}}{c^{2} m^{2}}},
$$

for a relativistic particle. The entropy $H$ corresponds to the classical Boltzmann-Maxwell entropy (of non quantum particles) when $\tau=0$, it corresponds to the Bose-Einstein entropy (of quantum particles of Bose type) when $\tau>0$ (and for the sake of simplicity we will restrict ourself to $\tau=1$, in the sequel) and it corresonds to the Fermi-Dirac entropy (of quantum particles of Fermi type) when $\tau<0$ (and again, we only consider the case $\tau=-1$ ).

Considering one of the above entropies $H$ and one of the above energies $\mathcal{E}$ we are therefore looking for a density function $\mathcal{G} \geq 0$ satisfying the moments constraint (1.2) and

$$
H(\mathcal{G})=\max _{g \text { satisfying }(1.2)} H(g) .
$$

The above entropy maximisation problem is a very fundamental problem of statistical physic since its solution $\mathcal{G}$ corresponds to the microscopic momentum distribution of a gas of particles at the rest whose macroscopic observable mass, momentum and energy are $N, P$ and $E$. The density distribution $\mathcal{G}$ is called the thermal equilibrium state. Out of rest, the evolution of the momentum gas distribution is usually discribed by a Boltzmann equation. The equilibrium state $\mathcal{G}$ is then (at least formally) a steady state to the associated Boltzmann equation. Moreover, any solution to the Boltzmann equation associated to an initial datum of macroscopic mass $N$, momentum $P$ and energy $E$ is expected 
to converge to the corresponding equilibrium state $\mathcal{G}$ in the large time asymptotic. For more details on this huge and difficult subject, we refer to [4], [22] and the many references therein for the classical Boltzmann equation, to [7], [18], [19] for the Fermi-Boltzmann equation, to [17], [11] for the Boltzmann equation associated to a gas of Bose particles and to [14], [8], [15], [1] for the relativistic Boltzmann equation. We also refer to [12] for a general mathematical presentation of the Boltzmann equation in a quantum and relativistic framework. A classical physical reference is [16].

A first simple and heuristic remark is that if $\bar{g}$ solves the entropy maximisation problem with constraint (1.2), there exists Lagrange multipliers $\mu \in \mathbb{R}$, $\beta^{0} \in \mathbb{R}$ and $\beta \in \mathbb{R}^{3}$ such that

$$
\langle\nabla H(\bar{g}), \varphi\rangle=\int_{\mathbb{R}^{3}} h^{\prime}(\bar{g}) \varphi \mathrm{d} p=\left\langle\beta^{0} \mathcal{E}(p)-\beta \cdot p-\mu, \varphi\right\rangle
$$

for all $\varphi$, which implies

$$
\ln (1+\tau \bar{g})-\ln \bar{g}=\beta^{0} \mathcal{E}(p)-\beta \cdot p-\mu,
$$

and in turn leads to

$$
\bar{g}(p)=\frac{1}{\mathrm{e}^{\nu(p)}-\tau} \quad \text { with } \quad \nu(p):=\beta^{0} \mathcal{E}(p)-\beta \cdot p-\mu .
$$

The function $\bar{g}$ is called a Maxwellian when $\tau=0$, a Bose-Einstein distribution when $\tau=1$ and a Fermi-Dirac distribution when $\tau=-1$.

Let us consider for a moment the case $\tau=0$, i.e. the classic (non-quantum non-relativistic) maximisation problem. In that case, the following result is known (and is almost trivial).

Theorem 1. - For any measurable function $\mathcal{G} \geq 0$ on $\mathbb{R}^{3}$ such that

$$
\int_{\mathbb{R}^{3}} \mathcal{G}\left(1, p, \frac{|p|^{2}}{2}\right) \mathrm{d} p=(N, P, E)
$$

for some $N, E>0, P \in \mathbb{R}^{3}$, the following assertions are equivalent:

(i) $\mathcal{G}$ is the Maxwellian

$$
\mathcal{M}_{N, P, E}=\mathcal{M}[\rho, u, \Theta]=\frac{\rho}{(2 \pi \Theta)^{3 / 2}} \exp \left(-\frac{|p-u|^{2}}{2 \Theta}\right)
$$

where $(\rho, u, \Theta)$ is uniquely determined by

$$
N=\rho, \quad P=\rho u, \quad E=\frac{\rho}{2}\left(|u|^{2}+3 \Theta\right) ;
$$

(ii) $\mathcal{G}$ is the solution of the maximisation problem

$$
H(\mathcal{G})=\max \{H(g) ; g \text { satisfies the moments constraint }(1.2)\},
$$

where $H(g)=-\int_{\mathbb{R}^{3}} g \log g \mathrm{~d} p$ stands for the classical entropy. 
Our main result is the extension of Theorem 1 to the quantum nonrelativistic and quantum relativistic framework, or in other words, we solve the maximisation problem (1.1)-(1.5) in the most general case. Before stating it, we would like to make some elementary remarks to convince the reader how different are the non quantum, the Bose and the Fermi cases.

On the one hand, the natural functional spaces to look for the density $f$ are the spaces of distribution $f \geq 0$ such that the "physical" quantities are bounded:

$$
\int_{\mathbb{R}^{3}} f(1+\mathcal{E}(p)) \mathrm{d} p<\infty \quad \text { and } \quad H(f)<\infty .
$$

In the Fermi case where $\tau=-1, h(f)=+\infty$ if $f \notin[0,1]$ and so $H(f)<\infty$ provides a strong $L^{\infty}$ bound on $f$. While in the Bose case, i.e. for $\tau=1$, one has $h(f) \sim \ln f$ when $f \rightarrow \infty$, so that the entropy bound does not give any additional information than the moments bound. This provides very different conditions since we obtain:

$$
f \in \begin{cases}L_{s}^{1} \cap L \log L & \text { in non quantum case, relativistic or not, } \\ L_{s}^{1} \cap L^{\infty} & \text { in the Fermi case, relativistic or not, } \\ L_{s}^{1} & \text { in the Bose case, relativistic or not, }\end{cases}
$$

where

$$
\left.L_{s}^{1}=\left\{f \in L^{1}\left(\mathbb{R}^{3}\right) ; \int_{\mathbb{R}^{3}}\left(1+|p|^{s}\right)|f(p)| \mathrm{d} p<\infty\right\}\right\},
$$

and $s=2$ in the non relativistic case, $s=1$ in the relativistic case.

On the other hand, it was already observed by Bose and Einstein (see [2], [9], [10]) that for systems of Bose particles in thermal equilibrium a careful analysis of the statistical physics of the problem leads to enlarge the class of steady distributions to include also the solutions containing a Dirac mass. More precisely, the class of Bose distributions $\bar{g}$ given by (1.6) has to be enlarged to the class of generalized Bose-Einstein relativistic distributions $\mathcal{B}$ defined by

$$
\mathcal{B}(p)=b+\alpha \delta_{p_{M C}}=\frac{1}{\mathrm{e}^{\nu(p)}-1}+\alpha \delta_{p_{M C}}, \quad \alpha \geq 0, p_{M C} \in \mathbb{R}^{3} .
$$

Moreover, and still concerning the Bose case, considering any fixed vector $a \in \mathbb{R}^{3}$ and any approximation of the identity $\left(\varphi_{n}\right)$ centered in $a$, it is shown in [3], see also Lemma 2.0, that for any $f \in L_{2}^{1}$ the quantity $H\left(f+\varphi_{n}\right)$ is well defined and

$$
\lim N\left(f+\alpha \varphi_{n}\right)=N(f)+\alpha \quad \text { and } \quad \lim H\left(f+\varphi_{n}\right)=H(f) \quad \text { as } n \rightarrow \infty .
$$

This indicates that the entropy $H$ may be extended to nonnegative measures and that, moreover, the singular part of the measure does not contributes to the entropy. We will come back to this question in Section 3 below.

In the Fermi case, the strong uniform bound entailed by the entropy on the Fermi distributions leads to include in the family of Fermi steady states the so 
called-degenerate states. Therefore, one has to enlarge the class of Fermi-Dirac states given by (1.6) to the class of distributions (see for instance [21])

$$
\mathcal{F}(p)=\frac{1}{\mathrm{e}^{\nu(p)}+1} \quad \text { and } \quad \chi(p)=\mathbf{1}_{\left\{\beta^{0} \mathcal{E}(p)-\beta \cdot p \leq 1\right\}} .
$$

Our main result reads as follows.

TheOREM 2. - For every possible choice of $(N, P, E)$ such that the set

$$
K=\left\{g ; \int_{\mathbb{R}^{3}} g(1, p, \mathcal{E}(p)) \mathrm{d} p=(N, P, E)\right\},
$$

is non empty, there exists a unique solution $\mathcal{G}$ to the entropy maximisation problem

$$
\mathcal{G} \in K, \quad H(\mathcal{G})=\max \{H(g) ; g \in K\} .
$$

Moreover, $\mathcal{G}$ is the unique thermal equilibrium, i.e. $\mathcal{G}=\bar{g}$ given by $(1.6)$ in the nonquantum case, $\mathcal{G}=\mathcal{B}$ given (1.8) in the Bose case, and $\mathcal{G}=\mathcal{F}$ given (1.9) in the Fermi case, satisfying the moments constraint (1.2).

We refer to Theorems 2.1, 3.2 and 4.1 for more precise statements. It is of course looked as an evidence, in the physicist community, that equilibrium states (1.6), (1.8) and (1.9) are the solution to the associated entropy maximisation problem. Nevertheless, in the quantum case, we were not able to find a convincing proof of this fact. Indeed, it is not clear at all how to obtain an explicit expression of the thermal equilibrium $\mathcal{G}$ (i.e. values of $\beta^{0}, \beta, \mu, \ldots$ ) as a function of the macropic quantities $N, P, E$. Our aim is to give here a rigorous and detailed proof of it.

The paper is organized as follows. In Section 2 we treat the relativistic non quantum case. In fact, this case was completely solved by R. Glassey and W.A. Strauss in [14], see also R. Glassey in [13] and [5]. However, we present here another proof, which uses in a crucial way, the Lorentz invariance and may be adapted to the quantum relativistic case.

We then deal with the Bose-Enstein gas in Section 3 and with the case of a Fermi-Dirac gas in Section 4. For each of these two kinds of gases, we first consider in detail the relativistic case and then briefly the non relativistic case, which is simplest since, by Galilean invariance, it can be reduced to $P=0$.

Acknowledgments. - We would like to thank A. Chambolle for useful discussions on maximisation problems and J.J.L. Velazquez for his encouragement and helpful comments during the elaboration of this work.

\section{Relativistic non-quantum gas}

In this section, we consider the Maxwell-Boltzmann entropy

$$
H(g)=-\int_{\mathbb{R}^{3}} g \ln g \mathrm{~d} p,
$$

BULlETIN DE LA SOCIÉTÉ MATHÉMATIQUE DE FRANCE 
of a non quantum gas. From the heuristic argument presented in the introduction, the solution to (1.2)-(1.5) is expected to be a relativistic Maxwellian distribution, which means that it is expected to be of the form

$$
\mathcal{M}(p)=\mathrm{e}^{-\beta^{0} p^{0}+\beta \cdot p-\mu},
$$

where we introduce the notation

$$
p^{0}=\mathcal{E}(p)=c \sqrt{c^{2} m^{2}+|p|^{2}}, \quad p \in \mathbb{R}^{3} .
$$

Our result is the following.

Theorem 2.1. - (i) Given $E, N>0, P \in \mathbb{R}^{3}$, there exists a least one function $g \geq 0$ which realizes the moments equation (1.2), (1.4) if, and only if,

$$
m^{2} c^{2} N^{2}+|P|^{2}<E^{2} .
$$

When (2.3) holds we will say that $(N, P, E)$ is admissible.

(ii) For any admissible $(N, P, E)$ there exists at least one relativistic Maxwellian distribution $\mathcal{M}$ given by (2.2) corresponding to these moments, i.e. satisfying (1.2).

(iii) Let $\mathcal{M}$ be a relativistic Maxwellian distribution. For any function $g \geq 0$ with the same moments, i.e. satisfying

$$
\int_{\mathbb{R}^{3}}\left(\begin{array}{c}
1 \\
p \\
p^{0}
\end{array}\right) g(p) \mathrm{d} p=\int_{\mathbb{R}^{3}}\left(\begin{array}{c}
1 \\
p \\
p^{0}
\end{array}\right) \mathcal{M}(p) \mathrm{d} p,
$$

one has

$$
H(g)-H(\mathcal{M})=H(g \mid \mathcal{M}):=\int_{\mathbb{R}^{3}}\left[g \ln \frac{g}{\mathcal{M}}-g+\mathcal{M}\right] \mathrm{d} p .
$$

Moreover, $H(g \mid \mathcal{M}) \leq 0$ and vanishes if, and only if, $g=\mathcal{M}$.

(iv) As a conclusion, for any admissible $(N, P, E)$, the entropy problem (1.2)-(1.5), (2.3)-(2.1) has a unique solution, and this one is the relativistic Maxwellian constructed just above.

Remark 2.2. - The relativistic Maxwell distribution $\mathcal{M}$ belongs to $L^{1}\left(\mathbb{R}^{3}\right)$ if, and only if, $\beta^{0}>0$ and $|\beta|<\beta^{0}$. In this case, all the moments of $\mathcal{M}$ are well defined and $\mathcal{M}$ takes its maximum at the point $p_{M C}$ such that

$$
\frac{p_{M C}}{p_{M C}^{0}}=\frac{\beta}{\beta^{0}}, \quad \text { and thus } \quad p_{M C}:=\frac{m c \beta}{\sqrt{\beta^{02}-|\beta|^{2}}} .
$$

We do not prove this claim, since we do not need it in the sequel and its proof is the same as that of Lemma 3.1 that we present in the next subsection.

We split the proof of Theorem 2.1 in three parts corresponding to the three first intermediary results (assertions (i), (ii), (iii) of Theorem 2.1). The last and main result (assertions (iv) of Theorem 2.1) is then an immediate consequence of the preceding ones. 
Proof of Theorem 2.1, (i). - Since $F(p) / N \mathrm{~d} p$ is a probability measure whose support is not a single point, and because the function $r \mapsto \mathcal{C}(r)=\sqrt{m^{2} c^{2}+r^{2}}$ is strictly convex, the Jensen inequality (which is therefore a strict inequality) implies

$$
\sqrt{m^{2} c^{2}+\left|\frac{P}{N}\right|^{2}}=\mathcal{C}\left(\int_{\mathbb{R}^{3}} p \frac{F(p)}{N \mathrm{~d} p}\right)<\int_{\mathbb{R}^{3}} \mathcal{C}(p) \frac{F(p)}{N \mathrm{~d} p}=\frac{E}{N}
$$

Proof of Theorem 2.1, (ii). - Given $(N, P, E)$ admissible, we look for a relativistic Maxwellian $\mathcal{M}$ (which means that we look for $\beta^{0}>0, \beta \in \mathbb{R}^{3}$ and $\mu \in \mathbb{R}$ ) such that

$$
N(\mathcal{M})=N, \quad P(\mathcal{M})=P, \quad E(\mathcal{M})=E .
$$

By symmetry the second equation implies that $\beta$ must be collinear to $P$. Therefore, in all the sequel, $P$ will denote the norm $|P|$ instead of a vector, so that the second equation as to be understood as a scalar equation.

The idea is now to reduce the system of three scalar equations (2.6) to a system of equations with less unknowns. A first way is to eliminate the $\mu$ dependence since the term $\mathrm{e}^{-\mu}$ can be factorised. This is the most classic way, which is used for instance by Glassey [13], but we do not follow it, since we do not know how to generalize it to the quantum case.

We begin introducing two new unknowns $(\bar{\beta}, u)$ with $\bar{\beta}>0, u \in \mathbb{R}^{3},|u|<c$, which correspond to the 4 -vector $\left(\beta^{0}, \beta\right) \in \mathbb{R}^{4}$ with $|\beta|<\beta^{0}$, by the following bijection

$$
u:=\frac{c \beta}{\beta^{0}}, \quad \bar{\beta}^{2}:=\left(\beta^{0}\right)^{2}-|\beta|^{2},
$$

and thus

$$
\beta^{0}=\gamma \bar{\beta}, \quad \beta=\frac{\gamma \bar{\beta} u}{c}, \quad \text { where } \quad \gamma:=\frac{1}{\sqrt{1-(u / c)^{2}}} .
$$

From the results recalled in the Appendix, we know that there is a Lorentz transformation $\Lambda_{u}$ associated to the velocity $u$ such that

$$
\left(\begin{array}{c}
\beta^{0} \\
\beta
\end{array}\right)=\Lambda_{u}\left(\begin{array}{c}
\bar{\beta} \\
0
\end{array}\right)
$$

In the initial frame, the identities (A.12), (A.13), (A.14) written for $\mathcal{M}$ give

$$
\left\{\begin{array}{l}
N(\mathcal{M})=A_{1} \gamma \bar{\beta}, \\
E(\mathcal{M})=A_{2}+A_{3}(\gamma \bar{\beta})^{2}, \\
P(\mathcal{M})=A_{3}(\gamma \bar{\beta})(\gamma \bar{\beta} u / c),
\end{array}\right.
$$

BULLETIN DE LA SOCIÉTÉ MATHÉMATIQUE DE FRANCE 
where

$$
\begin{array}{ll}
N(\phi)=\int_{\mathbb{R}^{3}} \phi \mathrm{d} p, & P(\phi)=\int_{\mathbb{R}^{3}} \phi p \mathrm{~d} p, \\
E(\phi)=\int_{\mathbb{R}^{3}} \phi p^{0} \mathrm{~d} p, & G(\phi)=m^{2} c^{2} \int_{\mathbb{R}^{3}} \phi \frac{\mathrm{d} p}{p^{0}},
\end{array}
$$

and where $A_{i}$ are invariant by change of frame under a Lorentz transform, see Lemma A.5.

Let us now introduce the Maxwellian $\overline{\mathcal{M}}$ in the rest frame, i.e. we define

$$
\overline{\mathcal{M}}(p)=\mathrm{e}^{-\bar{\beta} p^{0}-\mu} .
$$

In the rest frame, identities (A.12), (A.13), (A.15) written for $\overline{\mathcal{M}}$ are

$$
\left\{\begin{array}{l}
N(\overline{\mathcal{M}})=A_{1} \bar{\beta} \\
E(\overline{\mathcal{M}})=A_{2}+A_{3} \bar{\beta}^{2} \\
G(\overline{\mathcal{M}})=4 A_{2}+A_{3} \bar{\beta}^{2}
\end{array}\right.
$$

Inverting the two systems, we get

$$
\left\{\begin{array} { l } 
{ A _ { 1 } \overline { \beta } = N ( \mathcal { M } ) / \gamma } \\
{ A _ { 2 } = E ( \mathcal { M } ) - P ( \mathcal { M } ) c / u , } \\
{ A _ { 2 } + A _ { 3 } \overline { \beta } ^ { 2 } = E ( \mathcal { M } ) - P ( \mathcal { M } ) u / c }
\end{array} \quad \text { and } \quad \left\{\begin{array}{l}
A_{1} \bar{\beta}=N(\overline{\mathcal{M}}) \\
A_{2}=-H(\overline{\mathcal{M}}), \\
A_{2}+A_{3} \bar{\beta}^{2}=E(\overline{\mathcal{M}})
\end{array}\right.\right.
$$

where we have defined

$$
H(\phi):=\frac{1}{3}(E(\phi)-G(\phi))=\int_{\mathbb{R}^{3}} \phi \frac{|p|^{2}}{3 p^{0}} \mathrm{~d} p .
$$

Therefore, the fundamental relation between the relativistic Maxwellian $\mathcal{M}$ in the initial frame and the reduced relativistic Maxwellian $\overline{\mathcal{M}}$ in the rest frame is

$$
\left\{\begin{array}{l}
N(\mathcal{M})=N(\overline{\mathcal{M}}) \gamma(u) \\
P(\mathcal{M}) c / u-E(\mathcal{M})=H(\overline{\mathcal{M}}) \\
E(\mathcal{M})-P(\mathcal{M}) u / c=E(\overline{\mathcal{M}})
\end{array}\right.
$$

To find $\mathcal{M}$ such that (2.6) holds is therefore equivalent to find $\bar{\beta}>0, \mu \in \mathbb{R}$ and $u \geq 0$ such that

$$
\begin{cases}E=E(\bar{\beta}, \mu)+P u / c & \left(=: \Sigma_{E}(\bar{\beta}, \mu)\right), \\ N=N(\bar{\beta}, \mu) \gamma(u) & \left(=: \Sigma_{N}(\bar{\beta}, \mu)\right),\end{cases}
$$

where $u$ is given by

$$
u=u(\bar{\beta}, \mu):=\frac{P c}{E+H(\bar{\beta}, \mu)},
$$

and where we have defined

$$
L(\bar{\beta}, \mu):=L(\overline{\mathcal{M}})=L\left(\mathrm{e}^{-\bar{\beta} p^{0}}\right) \mathrm{e}^{-\mu}
$$


for $L=N, E, G, H$.

Existence of a solution to $(2.13),(2.14)$ is given by the following result which we state below and prove at the end of the section.

LEMmA 2.3. - 1) For all $\mu \in \mathbb{R}$ the function $\Sigma_{E}(., \mu): \mathbb{R}_{+}^{\star} \rightarrow \mathbb{R}$ is continuous, decreasing and such that

$$
\lim _{\bar{\beta} \rightarrow 0} \Sigma_{E}(\bar{\beta}, \mu)=+\infty \quad \text { and } \quad \lim _{\bar{\beta} \rightarrow+\infty} \Sigma_{E}(\bar{\beta}, \mu)=0 .
$$

Therefore, there exists a unique $\bar{\beta}=\bar{\beta}(\mu)>0$ such that $\Sigma_{E}(\bar{\beta}, \mu)=E$ for any $\mu$.

2) The function $\bar{\beta}: \mathbb{R} \rightarrow \mathbb{R}$ is continuous, decreasing,

$$
\lim _{\mu \rightarrow-\infty} \bar{\beta}(\mu)=+\infty \quad \text { and } \quad \lim _{\mu \rightarrow+\infty} \bar{\beta}(\mu)=0 .
$$

3) The function $\bar{\Sigma}_{N}: \mathbb{R} \rightarrow \mathbb{R}, \bar{\Sigma}_{N}(\mu):=\Sigma_{N}(\bar{\beta}(\mu), \mu)$ is continuous and decreasing. Moreover,

$$
\lim _{\mu \rightarrow-\infty} \bar{\Sigma}_{N}(\mu)=\sqrt{E^{2}-P^{2}} / m c \quad \text { and } \quad \lim _{\mu \rightarrow+\infty} \bar{\Sigma}_{N}(\mu)=0 .
$$

In particular, under the admissible condition $(2.3)$, there exists $\mu^{\star} \in \mathbb{R}$ such that $\bar{\Sigma}\left(\mu^{\star}\right)=N$.

As a conclusion, setting $\mu=\mu^{\star}, \bar{\beta}=\bar{\beta}\left(\mu^{\star}\right), u=u\left(\bar{\beta}\left(\mu^{\star}\right), \mu^{\star}\right)$, we have constructed a solution $(\bar{\beta}, \mu, u)$ to $(2.13)$ and $(2.14)$.

Assertion 3) of Lemma 2.3 states precisely that there exists a relativistic Maxwellian $\mathcal{M}$ satisfying (2.6), and this concludes the proof of assertion (ii) of Theorem 2.1.

Proof of Theorem 2.1, (iii). — Since $\ln \mathcal{M}=-\beta^{0} p^{0}+\beta \cdot p-\mu$ and using the moments equation (2.4), we get

$$
\begin{aligned}
H(g \mid \mathcal{M}) & =-\int_{\mathbb{R}^{3}} g \ln g \mathrm{~d} p+\int_{\mathbb{R}^{3}} g \ln \mathcal{M} \mathrm{d} p+\int_{\mathbb{R}^{3}}(\mathcal{M}-g) \mathrm{d} p \\
& =-\int_{\mathbb{R}^{3}} g \ln g \mathrm{~d} p+\int_{\mathbb{R}^{3}} \mathcal{M} \ln \mathcal{M} \mathrm{d} p=H(g)-H(\mathcal{M}) .
\end{aligned}
$$

Furthermore, the function $h_{s}(t):=t \ln (t / s)+s-t$ satisfies $h_{s}^{\prime}(t)=\ln (t / s)$ and $h_{s}^{\prime \prime}(t)=1 / t$ so that $h_{s}$ is strictly concave and $h_{s}(t)<h_{s}(s)=0$ for any $t \neq s$.

Proof of Lemma 2.3, (1). - For $L=N, E, G, H$ the function $L: \mathbb{R}_{+}^{*} \rightarrow \mathbb{R}_{+}^{*}$, $\bar{\beta} \mapsto L(\bar{\beta})$ is $\mathcal{C}^{1}$, decreasing, $\lim L(\bar{\beta})=+\infty$ when $\bar{\beta} \rightarrow 0$ and $\lim L(\bar{\beta})=0$ when $\bar{\beta} \rightarrow+\infty$. Then, $u(\bar{\beta}, \mu): \mathbb{R}_{+}^{\star} \times \mathbb{R} \rightarrow \mathbb{R}$ is continuous, increasing with respect to the two variables, $\lim u(\bar{\beta}, \mu)=0$ when $\bar{\beta} \rightarrow 0$ (for fixed $\mu$ ) and when $\mu \rightarrow-\infty$ (for fixed $\bar{\beta}), \lim u(\bar{\beta}, \mu)=c P / E(<c)$ when $\bar{\beta} \rightarrow+\infty$ (for fixed $\mu$ ) 
and when $\mu \rightarrow+\infty$ (for fixed $\bar{\beta})$. Last, $\gamma(\bar{\beta}, \mu):=\gamma(u(\bar{\beta}, \mu)): \mathbb{R}_{+}^{\star} \times \mathbb{R} \rightarrow \mathbb{R}$ is continuous, increasing with respect to the two variables, $\lim \gamma(\bar{\beta}, \mu)=1$ when $\bar{\beta} \rightarrow 0$ (for fixed $\mu$ ) and when $\mu \rightarrow-\infty$ (for fixed $\bar{\beta}$ ), $\lim \gamma(\bar{\beta}, \mu)=\gamma(c P / E)$ $(<+\infty)$ when $\bar{\beta} \rightarrow+\infty$ (for fixed $\mu)$ and when $\mu \rightarrow+\infty$ (for fixed $\bar{\beta})$.

We come now to the study of the function $\Sigma_{E}$. We clearly have $\Sigma_{E}: \mathbb{R}_{+}^{\star} \times$ $\mathbb{R} \rightarrow \mathbb{R}$ is $\mathcal{C}^{1}$ and the derivate $\Sigma_{E}^{\prime}$ with respect to the variables $\bar{\beta}$ or $\mu$ satisfies

$$
\begin{aligned}
\Sigma_{E}^{\prime}(\bar{\beta}, \mu) & =\frac{P}{c} u^{\prime}(\bar{\beta}, \mu)+E^{\prime}(\bar{\beta}, \mu) \\
= & \frac{P}{c}(-P c) \frac{H^{\prime}(\bar{\beta}, \mu)}{(E+H(\bar{\beta}, \mu))^{2}}+E^{\prime}(\bar{\beta}, \mu) \\
= & \left(\frac{u(\bar{\beta}, \mu)}{c}\right)^{2}\left[G^{\prime}(\bar{\beta}, \mu)+E^{\prime}(\bar{\beta}, \mu)\left(\left(\frac{E}{P}+\frac{H(\bar{\beta}, \mu)}{P}\right)^{2}-\frac{1}{3}\right)\right]<0
\end{aligned}
$$

since $E / P>1, E^{\prime}(\bar{\beta}, \mu)<0$ and $G^{\prime}(\bar{\beta}, \mu)<0$. This implies that $\Sigma_{E}$ is decreasing in both $\bar{\beta}$ and $\mu$. Moreover $\lim \Sigma_{E}(\bar{\beta}, \mu)=+\infty$ when $\bar{\beta} \rightarrow 0$ (for fixed $\mu$ ) or when $\mu \rightarrow-\infty$ (for fixed $\bar{\beta}), \lim \Sigma_{E}(\bar{\beta}, \mu)=P^{2} / E$ when $\bar{\beta} \rightarrow+\infty$ (for fixed $\mu$ ) or when $\mu \rightarrow+\infty$ (for fixed $\bar{\beta}$ ). Since $E>P$ and then $E>P^{2} / E$, for any $\mu \in \mathbb{R}$ there exists a unique $\bar{\beta}=\bar{\beta}(\mu)$ such that $\Sigma_{E}(\bar{\beta}, \mu)=E$.

Proof of Lemma 2.3, (2). - By the implicit function theorem, we know that $\mu \mapsto \bar{\beta}(\mu)$ is $\mathcal{C}^{1}$ and moreover

$$
\bar{\beta}^{\prime}(\mu)=-\frac{\partial \Sigma_{E} / \partial \mu}{\partial \Sigma_{E} / \partial \bar{\beta}}<0,
$$

so that $\bar{\beta}$ is decreasing. Assume by contradiction, that there is $\bar{\beta}_{\star}>0$ such that $\bar{\beta}(\mu) \geq \bar{\beta}_{\star}$ for any $\mu \in \mathbb{R}$. Then $L(\bar{\beta}(\mu), \mu) \leq L\left(\bar{\beta}_{\star}, \mu\right) \rightarrow 0$ when $\mu \rightarrow+\infty$ and therefore $\lim \Sigma_{E}(\bar{\beta}(\mu), \mu)=P^{2} / E<E$ which is in contradiction with the definition of $\bar{\beta}(\mu)$. Therefore $\lim \bar{\beta}(\mu)=0$ when $\mu \rightarrow+\infty$. Next, assume by contradiction that there is $\bar{\beta}^{\star}<+\infty$ such that $\bar{\beta}(\mu) \leq \bar{\beta}^{\star}$ for any $\mu \in \mathbb{R}$. Then $\Sigma_{E}(\bar{\beta}(\mu), \mu) \geq E(\bar{\beta}(\mu), \mu) \geq E\left(\bar{\beta}^{\star}, \mu\right) \rightarrow+\infty$ when $\mu \rightarrow-\infty$ which is again in contradiction with the definition of $\bar{\beta}(\mu)$. Therefore $\lim \bar{\beta}(\mu)=+\infty$ when $\mu \rightarrow-\infty$.

Proof of Lemma 2.3, (3). - It is clear that $\bar{\Sigma}_{N}$ is continuous as a composition of continuous functions. Since $|p| \leq p^{0} \leq|p|+m c$, we have the estimates

$$
\begin{gathered}
N(\bar{\beta}, \mu) \leq \mathrm{e}^{-\mu} \int_{\mathbb{R}^{3}} \mathrm{e}^{-\bar{\beta}|p|} \mathrm{d} p=C_{N} \frac{\mathrm{e}^{-\mu}}{\bar{\beta}^{3}}, \\
E(\bar{\beta}, \mu) \geq \mathrm{e}^{-\mu} \int_{\mathbb{R}^{3}}|p| \mathrm{e}^{-\bar{\beta}(|p|+m c)} \mathrm{d} p=C_{E} \frac{\mathrm{e}^{-\mu-\bar{\beta} m c}}{\bar{\beta}^{4}},
\end{gathered}
$$

TOME $133-2005-\mathrm{N}^{\mathrm{O}} 1$ 
where $C_{N}=\int_{\mathbb{R}^{3}} \mathrm{e}^{-|z|} \mathrm{d} z$ and $C_{E}=\int_{\mathbb{R}^{3}}|z| \mathrm{e}^{-|z|} \mathrm{d} z$. Then, using $E \geq E(\bar{\beta}(\mu), \mu)$ for any $\mu$, it yields

$$
\bar{\Sigma}_{N}(\mu) \leq C_{N} \frac{\mathrm{e}^{-\mu}}{\bar{\beta}^{3}} \gamma\left(\frac{c P}{E}\right) \leq E \frac{C_{N}}{C_{E}} \bar{\beta}(\mu) \mathrm{e}^{\bar{\beta} m c} \gamma\left(\frac{c P}{E}\right) \longrightarrow 0
$$

when $\mu \rightarrow+\infty$ (and thus $\lim \bar{\beta}(\mu)=0$ ).

We now claim that, there is $\alpha \geq 0$ and a sequence $\left(\mu_{n}\right)$ such that $\mu_{n} \rightarrow-\infty$ and

$$
\overline{\mathcal{M}}_{n}(p):=\mathrm{e}^{-\bar{\beta}\left(\mu_{n}\right) p^{0}-\mu_{n}} \underset{n \rightarrow+\infty}{\longrightarrow} \alpha \delta_{0}
$$

in the sense of measures, and in fact, most precisely $\left\langle\overline{\mathcal{M}}_{n}, \varphi\right\rangle \rightarrow \alpha \varphi(0)$ for any $\varphi \in C\left(\mathbb{R}^{3}\right)$ such that $\left(1+|p|^{-m}\right) \varphi(p) \rightarrow 0$ when $p \rightarrow \infty$ for some $m \geq 0$. Indeed, on one hand if for some $\bar{p} \in \mathbb{R}^{3} \backslash\{0\}$ we have $\mathcal{M}_{n}(2 \bar{p}) \geq \theta>0$ for any $n \geq 0$, then for any $p \in B(0,|\bar{p}|)$ we have

$$
\mathcal{M}_{n}(p)=\mathcal{M}(2 \bar{p}) \mathrm{e}^{\bar{\beta}\left(\mu_{n}\right)\left((2 \bar{p})^{0}-p^{0}\right)} \geq \theta \mathrm{e}^{\bar{\beta}\left(\mu_{n}\right)\left((2 \bar{p})^{0}-\bar{p}^{0}\right)} \longrightarrow+\infty
$$

so that $\lim E\left(\bar{\beta}\left(\mu_{n}\right), \mu_{n}\right)=+\infty$ which is in contradiction with the bound $E\left(\bar{\beta}\left(\mu_{n}\right), \mu_{n}\right) \leq E$. Therefore, $\lim \overline{\mathcal{M}}_{n}(p)=0$ when $n \rightarrow+\infty$ for any $p \in \mathbb{R}^{3} \backslash\{0\}$. On the other hand, fix $\bar{p} \in \mathbb{R}^{3} \backslash\{0\}$. We have already proved that there is some $\Theta$ such that $\mathcal{M}_{n}(\bar{p}) \leq \Theta$ for any $n \geq 0$. Therefore, for any $p \in \mathbb{R}^{3}$ such that $|p|>|\bar{p}|$ we have

$$
\mathcal{M}_{n}(p)=\mathcal{M}(\bar{p}) \mathrm{e}^{-\bar{\beta}\left(\mu_{n}\right)\left(p^{0}-\bar{p}^{0}\right)} \leq \Theta \mathrm{e}^{-\left(p^{0}-\bar{p}^{0}\right)} .
$$

Combining this with the bound $E\left(\bar{\beta}\left(\mu_{n}\right), \mu_{n}\right) \leq E$, we get (2.14) and in particular

$$
\lim N\left(\bar{\beta}\left(\mu_{n}\right), \mu_{n}\right)=\alpha, \quad \lim H\left(\bar{\beta}\left(\mu_{n}\right), \mu_{n}\right)=0, \quad \lim E\left(\bar{\beta}\left(\mu_{n}\right), \mu_{n}\right)=m c \alpha .
$$

Now, passing to the limit in (2.11)-(2.12) we get $E=m c \alpha+P^{2} / E$ so that

$$
\lim _{\mu \rightarrow-\infty} \bar{\Sigma}_{N}(\mu)=\alpha \gamma\left(\frac{P c}{E}\right)=\frac{E^{2}-P^{2}}{m c E} \frac{1}{\sqrt{1-(P / E)^{2}}}=\frac{\sqrt{E^{2}-P^{2}}}{m c} .
$$

\section{Bose gas}

We consider in this section the case of Bose particles. Let us start with the proof of the following remark, already stated in the introduction.

Lemma 3.0. - Let $a \in \mathbb{R}^{3}$ be any fixed vector and $\left(\varphi_{n}\right)_{n \in \mathbb{N}}$ an approximation of the identity:

$\left(\varphi_{n}\right)_{n \in \mathbb{N}}$ is a non negative sequence of $\mathcal{D}\left(\mathbb{R}^{3}\right)$ such that $\varphi_{n} \rightarrow \delta_{a}$ in $\mathcal{D}^{\prime}\left(\mathbb{R}^{3}\right)$.

BULLETIN DE LA SOCIÉTÉ MATHÉMATIQUE DE FRANCE 
For any $f \in L_{2}^{1}$ and any $n \in \mathbb{N}$, the quantity $H\left(f+\varphi_{n}\right)$ is well defined by (1.1), and

$$
\lim H\left(f+\varphi_{n}\right)=H(f) \quad \text { as } n \rightarrow \infty .
$$

Proof of Lemma 3.0. - Suppose indeed, for the sake of simplicity that $\varphi_{n} \equiv 0$ if $|p-a| \geq 2 / n$. Therefore:

$$
\begin{array}{rl}
H\left(f+\varphi_{n}\right)=\int_{|p-a| \geq 2 / n} & h(f(p, t), p) \mathrm{d} p \\
& +\int_{|p-a| \leq 2 / n} h\left(\left(f(p, t)+\varphi_{n}(p), p\right) \mathrm{d} p .\right.
\end{array}
$$

Since $|(1+z) \ln (1+z)-z \ln z| \leq c \sqrt{z}$

$$
\begin{aligned}
& \int_{|p-a| \leq 2 / n} \mid h(\left.f(p, t)+\varphi_{n}(p)\right) \mid \mathrm{d} p \\
& \leq c \frac{2}{\sqrt{n^{3}}}\left(\int_{|p-a| \leq 2 / n} h\left(f(p, t)+\varphi_{n}(p)\right) \mathrm{d} p\right)^{1 / 2} \longrightarrow 0 .
\end{aligned}
$$

Finally,

$$
\begin{array}{rl}
\mid \int_{\mathbb{R}^{3}} h(f(p, t), p) \mathrm{d} p-\int_{|p-a| \geq 2 / n} & h(f(p, t), p) \mathrm{d} p \mid \\
\leq \int_{|p-a| \leq 2 / n}|h(f(p, t), p)| \mathrm{d} p & \longrightarrow 0,
\end{array}
$$

which ends the proof.

Lemma 3.0 thus indicates that the expression of $H$ given in (1.1) may be extended to nonnegative measures and that, moreover, the singular part of the measure does not contributes to the entropy. We refer to [6] and [11] for a detailed proof of this claim. More precisely, for any non negative measure $F$ of the form $F=g \mathrm{~d} p+G$, where $g \geq 0$ is an integrable function = and $G \geq 0$ is singular with respect to the Lebesgue measure $d p$, we define the Bose-Einstein entropy of $F$ by

$$
H(F):=H(g)=\int_{\mathbb{R}^{3}}[(1+g) \ln (1+g)-g \ln g] \mathrm{d} p .
$$

On the other hand, the heuristic argument presented in the introduction has shown that the regular solution to the maximum entropy problem should be Bose relativistic distributions

$$
b(p)=\frac{1}{\mathrm{e}^{\nu(p)}-1} \quad \text { with } \quad \nu(p)=\beta^{0} p^{0}-\beta \cdot p+\mu .
$$

The following result explains simply where the Dirac masses have now to be placed.

TOME $133-2005-\mathrm{N}^{\mathrm{O}} 1$ 
Lemma 3.1. - The Bose relativistic distribution $b$ is non negative and belongs to $L^{1}\left(\mathbb{R}^{3}\right)$ if, and only if, $\beta^{0}>0,|\beta|<\beta^{0}$ and $\mu \geq \mu_{\bar{\beta}}$ with $\mu_{\bar{\beta}}:=-m c \bar{\beta}$, $\bar{\beta}>0$ and $\bar{\beta}^{2}=\left(\beta^{0}\right)^{2}-|\beta|^{2}$. In this case, all the moments of $b$ are well defined. Finally,

$$
\nu(p)>\nu\left(p_{M C}\right) \geq 0, \quad \forall p \neq p_{M C}:=\frac{m c \beta}{\sqrt{\beta^{02}-|\beta|^{2}}} .
$$

Proof of Lemma 3.1. - First, a necessary and sufficient condition for $b$ to be non negative is that $\nu(p) \geq 0$ for any $p \in \mathbb{R}^{3}$. Taking $p=t e$, where $e \in S^{2}$, $\beta=|\beta| e$ we see that $\lim \nu($ te $) / t=\beta^{0}-|\beta|$ when $t \rightarrow \infty$, and therefore $|\beta| \leq \beta^{0}$. We want now to prove that $b \in L^{1}\left(\mathbb{R}^{3}\right)$ implies that $\beta^{0}>0$ and $|\beta|<\beta^{0}$. The fact $\beta^{0}>0$ is obvious: in the contrary, $\beta=\beta^{0}=0$ and $b(p)$ is a positive constant, and thus do not belongs to $L^{1}\left(\mathbb{R}^{3}\right)$.

Let first assume, by contradiction, that

$$
\beta=\beta^{0} e, \quad e \in S^{2} \quad \text { and } \quad \beta^{0} m c+\mu=0 .
$$

Performing a spherical change of coordinates with $e$-axes, i.e. writing $p=$ er $\cos \theta+\cdots$, we obtain

$$
\begin{aligned}
N(b) & =\int_{\mathbb{R}^{3}} \frac{\mathrm{d} p}{\mathrm{e}^{\beta^{0}\left(p^{0}-p \cdot e\right)+\mu}-1}=2 \pi \int_{0}^{\infty} \int_{0}^{\pi} \frac{r^{2} \sin \theta \mathrm{d} \theta \mathrm{d} r}{\mathrm{e}^{\beta^{0}(\mathcal{E}(r)-r \cos \theta)+\mu}-1} \\
& \geq 2 \pi \int_{0}^{\infty} \int_{-1}^{1} \frac{r^{2} \mathrm{~d} r \mathrm{~d} t}{\mathrm{e}^{\beta^{0}(m c+r-t r)+\mu}-1} \geq 2 \pi \int_{0}^{\infty} \int_{0}^{2} \frac{r^{2} \mathrm{~d} r \mathrm{~d} s}{\mathrm{e}^{\beta^{0} r s} \mathrm{e}^{\beta^{0} m c+\mu}-1} \\
& \geq 2 \pi \int_{0}^{r_{0}} \int_{0}^{s_{0}} \frac{r^{2} \mathrm{~d} r \mathrm{~d} s}{2 \beta^{0} r s},
\end{aligned}
$$

choosing $s_{0}$ and $r_{0}$ small enough. Since the last expression is $+\infty$, we see that (3.4) can not hold.

Now assume, again by contradiction, that

$$
\beta=\beta^{0} e, \quad e \in S^{2} \quad \text { and } \quad \beta^{0} m c+\mu>0 .
$$

Writing $p=t e+q$ with $q \in \mathbb{R}^{3},|q| \leq 1$ and $q \perp e$ we compute

$$
\begin{aligned}
N(b) & =\int_{\mathbb{R}^{3}} \frac{\mathrm{d} p}{\mathrm{e}^{\beta^{0}\left(p^{0}-p \cdot e\right)+\mu}-1} \geq \int_{\mathbb{R}} \mathrm{d} t \int_{\mathbb{R}^{2}} \frac{\mathrm{d} q}{\mathrm{e}^{\beta^{0}\left(m c+\sqrt{t^{2}+|q|^{2}}-t\right)+\mu}-1} \\
& \geq 2 \pi \int_{\mathbb{R}} \frac{\mathrm{d} t}{\mathrm{e}^{\beta^{0}\left(\sqrt{t^{2}+1}-t\right)} \mathrm{e}^{\beta^{0} m c+\mu}-1} \geq 2 \pi \int_{t_{0}}^{+\infty} \frac{\mathrm{d} t}{\mathrm{e}^{\left(\beta^{0} m c+\mu\right) / 2}-1}
\end{aligned}
$$

choosing $t$ large enough. Once again the last expression is $+\infty$, so that (3.5) does not hold. Finally, since $\beta^{0} m c+\mu=\nu(0) \geq 0$, we must have $|\beta|<\beta^{0}$. From the preceding computations, we deduce that $\lim \nu(p)=+\infty$ when $|p| \rightarrow+\infty$ and therefore $\nu(p)$ reaches its minimum at a point $p_{M C}$ which satisfies

$$
\nabla_{p} \nu\left(p_{M C}\right)=\beta^{0} \frac{p_{M C}}{\sqrt{m^{2} c^{2}+\left|p_{M C}\right|^{2}}}-\beta=0 .
$$

BULLETIN DE LA SOCIÉTÉ MATHÉMATIQUE DE FRANCE 
The unique solution of this equation is $p_{M C}$ given in (3.3). As a conclusion, we get $\nu(p)>\nu\left(p_{M C}\right)$ for any $p \neq p_{M C}$ and the condition $\nu\left(p_{M C}\right) \geq 0$ implies $\mu \geq-m c \bar{\beta}$.

We define now the generalized Bose-Einstein relativistic distribution $\mathcal{B}$ by

$$
\mathcal{B}(p)=b+\alpha \delta_{p_{M C}}=\frac{1}{\mathrm{e}^{\nu(p)}-1}+\alpha \delta_{p_{M C}}
$$

with

$$
\forall p \neq p_{M C}, \quad \nu(p)=\beta^{0} p_{0}-\beta \cdot p+\mu>\nu\left(p_{M C}\right) \geq 0
$$

and the condition $\alpha \nu\left(p_{M C}\right)=0$.

For the Bose case, our main result reads as follows.

Theorem 3.2. - 1) Given $E, N>0, P \in \mathbb{R}^{3}$, there exists at least one measure $F \geq 0$ which realizes the moments equation (1.2) if, and only if,

$$
m^{2} c^{2} N^{2}+|P|^{2} \leq E^{2} .
$$

When (3.8) holds we will say that $(N, P, E)$ is admissible.

2) For any admissible $(N, P, E)$ there exists at least one relativistic BoseEinstein distribution $\mathcal{B}$ corresponding to these moments, i.e. satisfying (1.2).

3) Let $\mathcal{B}$ be a relativistic Bose-Einstein distribution. For any measure $F \geq 0$ satisfying

$$
\int_{\mathbb{R}^{3}}\left(\begin{array}{c}
1 \\
p \\
p^{0}
\end{array}\right) \mathrm{d} F(p)=\int_{\mathbb{R}^{3}}\left(\begin{array}{c}
1 \\
p \\
p^{0}
\end{array}\right) \mathrm{d} \mathcal{B}(p),
$$

one has

$$
H(F)-H(\mathcal{B})=H_{1}(g \mid b)+H_{2}(G \mid b)
$$

where

$$
\left\{\begin{array}{l}
H_{1}(g \mid b):=\int_{\mathbb{R}^{3}}\left((1+g) \ln \frac{1+g}{1+b}-g \ln \frac{g}{b}\right) \mathrm{d} p, \\
H_{2}(G \mid b):=-\int_{\mathbb{R}^{3}} \nu(p) \mathrm{d} G(p) .
\end{array}\right.
$$

Moreover, $H(F \mid \mathcal{B}) \leq 0$ and vanishes if, and only if, $F=\mathcal{B}$. In particular, $H(F)<H(\mathcal{B})$ if $F \neq \mathcal{B}$.

4) As a conclusion, for any admissible $(N, P, E)$, the entropy problem (1.5), (3.1), (1.4) has a unique solution, and this one is the relativistic Bose-Einstein distribution constructed just before.

The proof of part 1) of Theorem 3.2 is the same as the proof of (i) in Theorem 2.1 except that the Jensen inequality may be not strict since we deal with measures which can be concentrated at a single point. We present the proof of parts 2) and 3) pointing out the main differences with respect to 
the proof of (ii) and (iii) of Theorem 2.1. Then point 4) is an immediate consequence of the previous steps.

Proof of Theorem 3.2, 2). - We use the same notations as in the proof of part (ii) in Theorem 2.1. Given $(N, P, E)$ admissible, we look for $\mathcal{B}=b+\alpha \delta_{p_{M C}}$ such that

$$
\left\{\begin{array}{l}
N=N(\mathcal{B})=N(b)+\alpha, \\
E=E(\mathcal{B})=E(b)+\alpha p_{m, c}^{0}, \\
P=P(\mathcal{B})=P(b)+\alpha p_{M C} .
\end{array}\right.
$$

We introduce again the variables $(\bar{\beta}, u)$ and the Bose-Einstein distribution in the rest frame

$$
\bar{b}(p)=\frac{1}{\mathrm{e}^{\bar{\beta} p^{0}+\mu}-1} .
$$

We easily verify that

$$
p_{M C}=m \gamma u, \quad p_{m, c}^{0}=m c \gamma .
$$

By (2.8), with $\mathcal{M}$ replaced by $b$ and $\overline{\mathcal{M}}$ replaced by $\bar{b}$, to find $\mathcal{B}$ such that (3.12) holds is equivalent to find $(\bar{\beta}, u, \mu, \alpha)$ which satisfies

$$
\left\{\begin{array}{l}
N=N(\bar{\beta}, \mu) \gamma+\alpha, \\
P c / u-E=H(\bar{\beta}, \mu), \\
E-P u / c-\alpha m c / \gamma=E(\bar{\beta}, \mu),
\end{array}\right.
$$

where now $L(\bar{\beta}, \mu)$ stands for $L(\bar{b})$. Setting

$$
u=u(\bar{\beta}, \mu):=\frac{c P}{E+H(\beta, \mu)}, \quad \gamma(\beta, \mu)=\gamma(u(\beta, \mu)),
$$

the system (3.13) is equivalent to

$$
\left\{\begin{array}{l}
E-\alpha m c / \gamma(\bar{\beta}, \mu)=E(\bar{\beta}, \mu)+P / c u(\bar{\beta}, \mu) \\
N-\alpha=N(\bar{\beta}, \mu) \gamma(\bar{\beta}, \mu)
\end{array}\right.
$$

Let us define the functions

$$
\left\{\begin{array}{l}
\Gamma_{E}(\bar{\beta}, \alpha):=\alpha m c / \gamma(\bar{\beta})+E(\bar{\beta})+P / c u(\bar{\beta}), \\
\Gamma_{N}(\bar{\beta}, \alpha):=N(\bar{\beta}) \gamma(\bar{\beta})+\alpha \\
\Sigma_{E}(\bar{\beta}, \mu):=E(\bar{\beta}, \mu)+P / c u(\bar{\beta}, \mu) \\
\Sigma_{N}(\bar{\beta}, \mu):=N(\bar{\beta}, \mu) \gamma(\bar{\beta}, \mu)
\end{array}\right.
$$

with the notation $L(\bar{\beta}):=L\left(\bar{\beta}, \mu_{\bar{\beta}}\right), \mu_{\bar{\beta}}=-m c \bar{\beta}$ as in Lemma 3.1, for any $L=N, E, G, H$. The properties of $\Gamma_{E}, \Sigma_{E}$ and $\Sigma_{N}$ are then summarized in the following Lemma.

BULLETIN DE LA SOCIÉTÉ MATHÉMATIQUE DE FRANCE 
Lemma 3.3. - 1) There is a continuous function

$$
\bar{\beta}_{\star}:[0, N] \longrightarrow(0,+\infty), \quad \alpha \longmapsto \bar{\beta}_{\star}(\alpha),
$$

such that for any $\alpha \in[0, N], \Gamma_{E}\left(\bar{\beta}_{\star}(\alpha), \alpha\right)=E$, and $\bar{\beta}_{\star}=\bar{\beta}_{\star}(\alpha)$ is the unique solution of $\Gamma_{E}\left(\bar{\beta}_{\star}, \alpha\right)=E$.

2) We set $\mu_{\star}:=\mu_{\bar{\beta}_{\star}(0)}=-m c \bar{\beta}_{\star}(0)$. There is a continuous function

$$
\bar{\beta}:\left[\mu_{\star},+\infty\right) \longrightarrow(0,+\infty), \quad \mu \longmapsto \bar{\beta}(\mu),
$$

such that $\Sigma_{E}(\bar{\beta}(\mu), \mu)=E$ for any $\mu \geq \mu_{\star}$, and $\bar{\beta}=\bar{\beta}(\mu)$ is the unique solution of the equation $\Sigma_{E}(\bar{\beta}, \mu)=E$. Moreover, the map $\bar{\beta}$ is not increasing and $\lim \bar{\beta}(\mu)=0$ when $\mu \rightarrow+\infty$. Last, the function

$$
\mu \longmapsto \bar{\Sigma}_{N}(\mu):=\Sigma_{N}(\bar{\beta}(\mu), \mu)
$$

is continuous on $\left[\mu_{\star},+\infty\right)$ and $\lim \bar{\Sigma}_{N}(\mu)=0$ when $\mu \rightarrow+\infty$.

Let us use Lemma 3.3 to conclude the proof of part 2) of Theorem 3.2. Its proof is postponed to the end of the proof of Theorem 3.2.

End of the proof of part 2) of Theorem 3.2. - In order to obtain the solution $(\bar{\beta}, u, \mu, \alpha)$ to $(3.13)$ we consider the two cases:

$$
N>\Gamma_{N}\left(\bar{\beta}_{\star}(0), 0\right) \quad \text { and } \quad N \leq \Gamma_{N}\left(\bar{\beta}_{\star}(0), 0\right) .
$$

- If $N>\Gamma_{N}\left(\bar{\beta}_{\star}(0), 0\right)$, we remark that

$$
\Gamma_{N}\left(\bar{\beta}_{\star}(N), N\right)=N\left(\bar{\beta}_{\star}(N)\right) \gamma\left(\bar{\beta}_{\star}(N)\right)+N>N .
$$

Therefore, by the intermediary value Theorem there exists $\left.\alpha^{\star} \in\right] 0, N[$ such that $\Gamma_{N}\left(\bar{\beta}_{\star}\left(\alpha^{\star}\right), \alpha^{\star}\right)=N$. Therefore, we define

$$
\bar{\beta}^{\star}:=\bar{\beta}_{\star}\left(\alpha^{\star}\right), \quad u^{\star}:=u\left(\bar{\beta}^{\star}\right), \quad \mu^{\star}:=0 .
$$

- If $N \leq \Gamma_{N}\left(\bar{\beta}_{\star}(0), 0\right)$, we remark that $\bar{\Sigma}_{N}\left(\mu_{\star}\right)=\Gamma_{N}\left(\bar{\beta}_{\star}(0), 0\right) \geq N$ and $\lim \bar{\Sigma}_{N}(\mu)=0$ when $\mu \rightarrow+\infty$, and by the intermediate value Theorem there exists $\mu^{\star} \in\left[\mu_{\star},+\infty\right)$ such that $\bar{\Sigma}_{N}\left(\mu^{\star}\right)=N$. We define now

$$
\bar{\beta}^{\star}:=\bar{\beta}_{E}\left(\mu^{\star}\right), \quad u^{\star}:=u\left(\bar{\beta}^{\star}, \mu^{\star}\right) \quad \alpha^{\star}:=0 .
$$

In both cases $\left(\beta^{\star}, \mu^{\star}, u^{\star}, \alpha^{\star}\right)$ is a solution of (3.13), and the associated relativistic Bose state is a solution of (3.12). 
Proof of part 3) of Theorem 3.2. - Using the conditions on the moments, we compute

$$
\begin{aligned}
H(b) & =\int_{\mathbb{R}^{3}} \ln (1+b) \mathrm{d} p+\int_{\mathbb{R}^{3}} b \ln \frac{1+b}{b} \mathrm{~d} p=\int_{\mathbb{R}^{3}} \ln (1+b) \mathrm{d} p+\int_{\mathbb{R}^{3}} b \nu(p) \mathrm{d} p \\
& =\int_{\mathbb{R}^{3}} \ln (1+b) \mathrm{d} p+\int_{\mathbb{R}^{3}}\left(g+G-\alpha \delta_{p_{M C}}\right) \nu(p) \mathrm{d} p \\
& =\int_{\mathbb{R}^{3}} \ln (1+b) \mathrm{d} p+\int_{\mathbb{R}^{3}} g \ln \frac{1+b}{b} \mathrm{~d} p+\int_{\mathbb{R}^{3}}\left(G-\alpha \delta_{p_{M C}}\right) \nu(p) \mathrm{d} p \\
& =\int_{\mathbb{R}^{3}}((1+g) \ln (1+b)-g \ln b) \mathrm{d} p+\int_{\mathbb{R}^{3}} \nu(p) \mathrm{d} G(p),
\end{aligned}
$$

from where we deduce (3.10),(3.11). On the other hand, for any $y>0$, the function $x \mapsto h(x \mid y)$ defined for any $x \geq 0$ by

$$
h(x \mid y):=(x+1) \ln \frac{x+1}{y+1}-x \ln \frac{x}{y},
$$

satisfies $h^{\prime}(x \mid y)=0$ if, and only if, $x=y, 1(y \mid y)=0$ and $h(x \mid y)<0$ for any $x \neq y$. Thus, $H(g \mid \mathcal{B}) \leq 0$ and $H(g \mid \mathcal{B})=0$ if and only if, $g=b$. Moreover, from (3.7), we obviously have $H(G \mid \mathcal{B}) \leq 0$ and $H(G \mid \mathcal{B})=0$ if, and only if, $G=\alpha \delta_{p_{M C}}$ with $\alpha=0$ if $\mu>0$ and $\alpha=m-M(b)$ if $\mu \geq 0$.

Proof of Lemma 3.3. - Let us prove point 1) of Lemma 3.3. To this end, consider the function $\Gamma_{E}(\bar{\beta}, \alpha)$. Notice first that for $L=N, E, G, H$ the function $L(\bar{\beta})$ is nothing but $L\left(\mathcal{P}_{\bar{\beta}}\right)$ where $\mathcal{P}_{\bar{\beta}}$ is the distribution

$$
\mathcal{P}_{\bar{\beta}}=\frac{1}{\mathrm{e}^{\bar{\beta}\left(p^{0}-m c\right)}-1} .
$$

Moreover, the maps $L=N, E, G, H$ are defined on the set

$$
\mathcal{O}=\left\{(\bar{\beta}, \mu) ; \bar{\beta}>0, \mu \geq \mu_{\bar{\beta}}\right\}=\left\{(\bar{\beta}, \mu) ; \mu \in \mathbb{R}, \bar{\beta} \geq \bar{\beta}_{\mu}=\max (0,-\mu / m c)\right\} .
$$

Since,

$$
L^{\prime}(\bar{\beta})=\frac{\mathrm{d} L(\bar{\beta})}{\mathrm{d} \bar{\beta}} \equiv \frac{\partial L}{\partial \bar{\beta}}-m c \frac{\partial L}{\partial \bar{\beta}},
$$

we obtain that the maps $\bar{\beta} \mapsto L(\bar{\beta})$ are $\mathcal{C}^{1}$, decreasing, $\lim L(\bar{\beta})=+\infty$ when $\bar{\beta} \rightarrow \bar{\beta}_{\mu}$ and $\lim L(\bar{\beta})=0$ when $\bar{\beta} \rightarrow+\infty$. Moreover for any fixed $\alpha$

$$
\frac{\partial \Gamma_{E}(\bar{\beta}, \alpha)}{\partial \bar{\beta}}=\left\{\frac{\alpha m c}{\gamma(\bar{\beta})}\right\}^{\prime}+E^{\prime}(\bar{\beta})+\frac{P}{c} u^{\prime}(\bar{\beta})
$$

A straightforward computation gives

$$
E^{\prime}(\bar{\beta})+\frac{P}{c} u^{\prime}(\bar{\beta})=\left(E_{\bar{\beta}}-m c E_{\mu}\right)\left(1-\frac{1}{3}\left(\frac{u(\bar{\beta})}{c}\right)^{2}\right)+\left(\frac{u(\bar{\beta})}{c}\right)^{2} \frac{G_{\bar{\beta}}-m c G_{\mu}}{3}<0
$$

BULlETIN DE LA SOCIÉTÉ MATHÉMATIQUE DE FRANCE 
and

$$
\left\{\frac{\alpha m c}{\gamma(\bar{\beta})}\right\}^{\prime}=\frac{\alpha m u^{3}(\bar{\beta})}{P c^{2}} \gamma^{-1}(\bar{\beta})\left(H_{\bar{\beta}}-m c H_{\mu}\right)<0 .
$$

We deduce that, for any fixed $\alpha \in[0, N]$, the map $\bar{\beta} \mapsto \Gamma_{E}(\bar{\beta}, \alpha)$ is $\mathcal{C}^{1}$, decreasing, $\lim \Gamma_{E}(\bar{\beta}, \alpha)=+\infty$ when $\bar{\beta} \rightarrow \bar{\beta}_{\mu}$ and

$$
\lim \Gamma_{E}(\bar{\beta}, \alpha)=\alpha \frac{m c}{\gamma(c P / E)}+\frac{P^{2}}{E} \quad \text { when } \quad \bar{\beta} \rightarrow+\infty .
$$

On the other hand, for any $\alpha \in[0, N]$, the admissibility condition (3.8) on $(N, P, E)$ gives

$$
\begin{aligned}
\left(\alpha \frac{m c}{\gamma(c P / E)}\right)^{2} & \leq\left(N m c \sqrt{1-(P / E)^{2}}\right)^{2} \\
& \leq\left(E^{2}-P^{2}\right)\left(1-\left(\frac{P}{E}\right)^{2}\right)=\left(E-\frac{P^{2}}{E}\right)^{2} .
\end{aligned}
$$

Therefore, we get $\lim \Gamma_{E}(\bar{\beta}, \alpha) \leq E$ when $\bar{\beta} \rightarrow+\infty$. Using once more the intermediate value Theorem, there exists a unique $\bar{\beta}_{\star}=\bar{\beta}_{\star}(\alpha) \geq \bar{\beta}_{\mu}$ such that $E=\Gamma_{E}\left(\bar{\beta}_{\star}(\alpha), \alpha\right)$. Finally, the function $\alpha \mapsto \bar{\beta}_{\star}(\alpha)$ is continuous (by the implicit function Theorem, for instance).

Let us prove now part 2). Here the proof is the same as that of Lemma 2.1. The only difference is that the maps $L$ are only defined on the set

$$
\mathcal{O}=\left\{(\bar{\beta}, \mu) ; \bar{\beta}>0, \mu \geq \mu_{\bar{\beta}}\right\}=\left\{(\bar{\beta}, \mu) ; \mu \in \mathbb{R}, \bar{\beta} \geq \bar{\beta}_{\mu}=\max (0,-\mu / m c)\right\} .
$$

Actually, we are only interested by

$$
\mathcal{O}_{\star}=\left\{(\bar{\beta}, \mu) ; \mu \geq \mu_{\star}, \bar{\beta} \geq \bar{\beta}_{\mu}\right\} .
$$

We prove without any difficulty that for any $\mu \geq \mu_{\star}$ and any function $L=$ $E, N, G$ and $H$, the functions $L(., \mu):\left(\bar{\beta}_{\mu},+\infty\right) \rightarrow(0,+\infty), \bar{\beta} \mapsto L(\bar{\beta}, \mu)$ are $\mathcal{C}^{1}$, decreasing, $\lim L(\bar{\beta}, \mu)=0$ when $\bar{\beta} \rightarrow+\infty$ and $\lim L(\bar{\beta}, \mu)=+\infty$ when $\bar{\beta} \rightarrow \bar{\beta}_{\mu}$. We deduce $\Sigma_{E}(., \mu):\left(\bar{\beta}_{\mu},+\infty\right) \rightarrow(0,+\infty)$ is $\mathcal{C}^{1}$, decreasing, $\lim \Sigma_{E}(\bar{\beta}, \mu)=+\infty$ when $\bar{\beta} \rightarrow \bar{\beta}_{\mu}$ and $\lim \Sigma_{E}(\bar{\beta}, \mu)=P^{2} / E \leq E$ when $\bar{\beta} \rightarrow+\infty$. As a consequence, for any $\mu \geq \mu_{\star}$, there exists a unique $\bar{\beta}=\bar{\beta}(\mu)$ such that $E=\Sigma_{E}\left(\bar{\beta}_{\star}(\mu), \mu\right)$.

On the other hand, one can also verify that for any $\bar{\beta}>0$ and any $L=E, N, G$ and $H, L(\bar{\beta},):.\left(\mu_{\star},+\infty\right) \rightarrow(0,+\infty)$ is $\mathcal{C}^{1}$, decreasing and $\lim L(\bar{\beta}, \mu)=0$ when $\mu \rightarrow+\infty$. Therefore, as in Lemma 2.3 , (ii), one checks that $\Sigma_{E}(\bar{\beta}, \mu)$ is $\mathcal{C}^{1}$ and decreasing in both variables. We deduce that the function $\mu \mapsto \bar{\beta}(\mu)$ is decreasing and $\lim \bar{\beta}(\mu)=0$ when $\bar{\beta} \rightarrow+\infty$. Finally, $\bar{\Sigma}_{N}$ is continuous and satisfies $\lim \bar{\Sigma}_{N}(\mu)=0$ when $\mu \rightarrow+\infty$. 
Nonrelativistic Bose particles. - For non relativistic particles the energy is $\mathcal{E}(p)=|p|^{2} / 2 m$. By Galilean invariance the problem (1.2) is then equivalent to the following simpler one: given three quantities $N>0, E>0, P \in \mathbb{R}^{3}$ find $F(p)$ such that

$$
\int_{\mathbb{R}^{3}}\left(\begin{array}{c}
1 \\
p-P / N \\
|p-P / N|^{2} / 2 m
\end{array}\right) F(p) \mathrm{d} p=\left(\begin{array}{c}
N \\
0 \\
E-|P|^{2} / 2 m N
\end{array}\right) .
$$

It is rather simple, using elementary calculus, to prove that for any $E, N>0$, $P \in \mathbb{R}^{3}$ there exists a distribution of the from

$$
F(p)=\frac{1}{\mathrm{e}^{a|p-P / N|^{2}+b^{+}}-1}-b^{-} \delta_{P / N}
$$

with $a \in \mathbb{R}, b \in \mathbb{R}, \nu \in \mathbb{R}, b^{+}=\max (b, 0), b^{-}=-\max (-b, 0)$ which satisfies (3.16).

Once such a solution (3.17) of (3.16) is obtained, the following Bose-Einstein distribution

$$
\mathcal{B}(p)=\frac{1}{\mathrm{e}^{\nu(p)}-1}+\alpha \delta_{p_{M C}}, \quad \nu(p)=a|p|^{2}-\frac{2 a}{N} p+\left(b^{+}+\frac{a|P|^{2}}{N^{2}}\right)
$$

solves (1.2).

This shows that for non relativistic particles Theorem 3.2 remains valid under the unique following change: points 1) and 2) have to be replaced by

$\left.1^{\prime}\right)$ For every $E, N>0, P \in \mathbb{R}^{3}$, there exists one relativistic Bose Einstein distribution defined by (3.17) corresponding to these moments, i.e. satisfying (1.2).

Points 3) and 4) of Theorem 3.2 remain unchanged.

It is particularly simple to observe in this case that, for $E>0$ and $P \in \mathbb{R}^{3}$ fixed, there is no regular Bose Einstein state for particle numbers $N$ larger than a critical value $N^{*}$. This easily follows from the fact that by (3.18),

$$
\begin{aligned}
E \geq & \int_{\mathbb{R}^{3}} \frac{|p|^{2}}{\mathrm{e}^{a|p-P / N|^{2}}-1} \mathrm{~d} p=\int_{\mathbb{R}^{3}} \frac{|q|^{2}}{\mathrm{e}^{a|q|^{2}}-1} \mathrm{~d} q+\frac{|P|^{2}}{N} \\
= & \frac{1}{a^{2}} \int_{\mathbb{R}^{3}} \frac{|p|^{2}}{\mathrm{e}^{|p|^{2}}-1} \mathrm{~d} p+\frac{|P|^{2}}{N} \\
& =\frac{1}{a^{5 / 2}} \int_{\mathbb{R}^{3}} \frac{|p|^{2}}{\mathrm{e}^{|p|^{2}}-1} \mathrm{~d} p+|P|^{2} a^{3 / 2}\left[\int_{\mathbb{R}^{3}} \frac{\mathrm{d} p}{\mathrm{e}^{|p|^{2}}-1}\right]^{-1} .
\end{aligned}
$$

In particular,

$$
a \geq\left[\frac{1}{E} \int_{\mathbb{R}^{3}} \frac{|p|^{2}}{\mathrm{e}^{|p|^{2}}-1} \mathrm{~d} p\right]^{2 / 5}=: a_{0}
$$

BULlETIN DE LA SOCIÉTÉ MATHÉMATIQUE DE FRANCE 
and therefore

$$
N=\int_{\mathbb{R}^{3}} \frac{\mathrm{d} p}{\mathrm{e}^{a|p-P / N|+b^{+}}-1} \leq \int_{\mathbb{R}^{3}} \frac{\mathrm{d} q}{\mathrm{e}^{a_{0}|q|^{2}}-1}=N^{*} .
$$

\section{Fermi-Dirac gas}

In this section we are interested in a Fermi-Dirac gas, which means that we consider the maximum entropy problem for the Fermi-Dirac entropy

$$
H_{F D}(f):=-\int_{\mathbb{R}^{3}}((1-f) \ln (1-f)+f \ln f) \mathrm{d} p .
$$

In particular, this implies the constraint $0 \leq f \leq 1$ on the density $f$ of the gas.

From the heuristics argument presented in the introduction, we know $=$ that the solution $\mathcal{F}$ of $(1.2)-(2.3),(4.1)$ is the Fermi-Dirac distribution

$$
\mathcal{F}(p)=\frac{1}{\mathrm{e}^{\nu(p)}+1} \quad \text { with } \quad \nu(p)=\beta^{0} p^{0}-\beta \cdot p+\mu .
$$

We also introduce the "saturated" Fermi-Dirac (SFD) density

$$
\chi(p)=\chi_{\beta^{0}, \beta}(p)=\mathbf{1}_{\left\{\beta^{0} p^{0}-=\beta \cdot p \leq 1\right\}}=\mathbf{1}_{\mathcal{E}}
$$

with $\mathcal{E}=\left\{\beta^{0} p^{0}-\beta \cdot p \leq 1\right\}, \beta \in \mathbb{R}^{3}$ and $\beta^{0}>|\beta|$.

Our main result is the following.

Theorem 4.1. - 1) For any $P$ and $E$ such that $|P|<E$ there exists an unique SFD state $\chi=\chi_{P, E}$ such that $P(\chi)=P$ and $E(\chi)=E$. This one realizes the maximum of particles number for given energy $E$ and mean momentum $P$. More precisely, for any $f$ such that $0 \leq f \leq 1$ one has

$$
P(f)=P, E(f)=E \quad \text { implies } \quad N(f) \leq N\left(\chi_{P, E}\right) .
$$

As a consequence, given $(N, P, E)$ there exists $g$ satisfying the moments equation (1.2) if, and only if, $E>|P|$ and $0 \leq N \leq N\left(\chi_{P, E}\right)$. In this case, we say that $(N, P, E)$ is admissible.

2) For any $(N, P, E)$ admissible there exists a Fermi-Dirac state $\mathcal{F}$ ("saturated" or not) which realizes the moments equation (1.2).

3) Let $\mathcal{F}$ be a Fermi-Dirac state. For any $f$ such that $0 \leq f \leq 1$ and

$$
\int_{\mathbb{R}^{3}} f(p)\left(\begin{array}{c}
1 \\
p \\
p^{0}
\end{array}\right) \mathrm{d} p=\int_{\mathbb{R}^{3}} \mathcal{F}(p)\left(\begin{array}{c}
1 \\
p \\
p^{0}
\end{array}\right) \mathrm{d} p,
$$

one has

$$
H_{F D}(f)-H_{F D}(\mathcal{F})=H_{F D}(f \mid \mathcal{F}):=\int_{\mathbb{R}^{3}}\left((1-f) \ln \frac{1-f}{1-\mathcal{F}}-f \ln \frac{f}{\mathcal{F}}\right) \mathrm{d} p .
$$


4) As a conclusion, for any admissible $(N, P, E)$ the entropy problem (1.5), (1.2), (4.1), (1.4) has a unique solution, and this one is the relativistic FermiDirac distribution constructed just above.

The new difficulty with respect to the classic or Bose case is to manage with the constraint $0 \leq f \leq 1$. Before proving Theorem 4.1 we present several auxiliary results.

Proposition 4.2. - 1) For any $P \in \mathbb{R}^{3}$ and $E>0$ such that $|P|<E$ there exists a unique SFD state $\chi=\chi_{P, E}$ such that $P(\chi)=P$ and $E(\chi)=E$. Moreover, for fixed $P \in \mathbb{R}^{3}$, the map $E \mapsto N\left(\chi_{P, E}\right)$ is increasing.

2) For any $N>0$ and $P \in \mathbb{R}^{3}$ there exists an unique $S F D$ state $\widetilde{\chi}=\widetilde{\chi}_{N, P}$ such that $N(\widetilde{\chi})=N$ and $P(\widetilde{\chi})=P$. Moreover, for fixed $P \in \mathbb{R}^{3}$, the map $N \mapsto E\left(\widetilde{\chi}_{N, P}\right)$ is increasing.

Proof. - We start with part 1). From (2.8) we know that for any SFD state $\chi$ of the form (4.3) we have

$$
P(\chi) c / u-E(\chi)=H(\bar{\beta}), \quad E(\chi)-P(\chi) u / c=E(\bar{\beta}), \quad N(\chi)=N(\bar{\beta}) \gamma
$$

where $L(\bar{\beta})=L(\bar{\chi}), \bar{\chi}(p)=\mathbf{1}_{\overline{\mathcal{E}}}, \overline{\mathcal{E}}=\left\{\bar{\beta} p^{0} \leq 1\right\}$ and $(\bar{\beta}, u)$ is associated to $\left(\beta^{0}, \beta\right)$ thanks to $(2.7)$.

On the other hand, from the two first equations in (4.6), to find $\chi$ such that, for given $P \in \mathbb{R}^{3}$ and $E>|P|^{2}, P(\chi)=P$ and $E(\chi)=E$ is equivalent to find $(\bar{\beta}, u)$ such that $\bar{\beta}$ satisfies

$$
E=\Xi(\bar{\beta}, E):=E(\bar{\beta})+\frac{P^{2}}{E+H(\bar{\beta})}
$$

and we recover $u$ by

$$
u=u(\bar{\beta}):=\frac{P c}{E+H(\bar{\beta})} .
$$

We have already done the analysis of such a function $\Xi$ in Lemma 2.1. It is easy to verify that $E(\bar{\beta})$ and $H(\bar{\beta})$ are smooth, decreasing for $\bar{\beta} \in(0,1 / m c)$, $\lim E(\bar{\beta})=\lim H(\bar{\beta})=0$ when $\bar{\beta} \rightarrow 1 / m c, \lim E(\bar{\beta})=\lim H(\bar{\beta})=\infty$ when $\bar{\beta} \rightarrow 0$ and

$$
\frac{\partial \Xi}{\partial \bar{\beta}}(\bar{\beta}, E)=E^{\prime}(\bar{\beta})-\frac{P^{2}}{(E+H(\bar{\beta}))^{2}} H^{\prime}(\bar{\beta})<0 .
$$

Moreover, $\lim \Xi(\bar{\beta}, E)=\infty$ when $\bar{\beta} \rightarrow 0$ (since $\Xi(\bar{\beta}, E) \geq E(\bar{\beta})$ ) and $\lim \Xi(\bar{\beta}, E)=P^{2} / E$ when $\bar{\beta} \rightarrow 1 / m c$. This implies that there is a unique $\bar{\beta} \in(0,1 / m c)$ such that (4.7) holds (when $|P|^{2}<E$ ), we note $\bar{\beta}=\bar{\beta}(E)$. Finally,

$$
\frac{\partial N\left(\chi_{P, E}\right)}{\partial E}=\frac{\mathrm{d} N(\bar{\beta}(E))}{\mathrm{d} E}=N^{\prime}(\bar{\beta}(E)) \frac{\mathrm{d} \bar{\beta}}{\mathrm{d} E}>0,
$$

BULlETIN DE LA SOCIÉTÉ MATHÉMATIQUE DE FRANCE 
since $N^{\prime}(\bar{\beta})<0$ and $\mathrm{d} \bar{\beta} / \mathrm{d} E<0$, and therefore $E \mapsto N\left(\chi_{P, E}\right)$ is inceassing (for fixed $P \in \mathbb{R}^{3}$ ).

Let us prove now part 2). We eliminate the energy $E(\chi)$ in (4.6) to get

$$
\frac{1}{\gamma^{2}} P(\chi) \frac{c}{u}=E(\bar{\beta})+H(\bar{\beta}), \quad N(\chi)=N(\bar{\beta}) \gamma .
$$

Therefore, for given $N>0$ and $P \in \mathbb{R}^{3}$, find $\chi$ such that $N(\chi)=N$ and $P(\chi)=P$ is equivalent to find $(\bar{\beta}, u)$ such that $\bar{\beta}$ satisfies

$$
N=N(\bar{\beta}) \gamma(u(\bar{\beta}))
$$

where $u$ is defined by

$$
u(\bar{\beta})=\frac{c P}{N^{2}} \cdot \frac{N^{2}(\bar{\beta})}{E(\bar{\beta})+H(\bar{\beta})} .
$$

In order to simplify the computations in what follow we introduce the variable $a=1 / \bar{\beta}$ and we define $\tilde{L}(a)=L(1 / \bar{\beta})$. We make the elementary computations

$$
\begin{array}{ll}
\widetilde{N}(a)=\frac{1}{3}\left(a^{2}-1\right)^{3 / 2}, & \widetilde{N}^{\prime}(a)=4 a \pi\left(a^{2}-m^{2} c^{2}\right)^{1 / 2}, \\
\widetilde{E}(a) \geq \frac{1}{4}\left(a^{2}-1\right)^{2}, & \widetilde{E}^{\prime}(a)=4 \pi a^{2}=\left(a^{2}-m^{2} c^{2}\right)^{1 / 2}, \\
\widetilde{H}(a) \geq 0, & \widetilde{G}^{\prime}(a)=4 \pi\left(a^{2}-m^{2} c^{2}\right)^{1 / 2} .
\end{array}
$$

We look now for a solution $a \in(1, \infty)$ to

$$
N=\Xi(a, N):=\tilde{N}(a) \gamma(\tilde{u}(a)),
$$

where the dependency of $\Xi$ with respect to $N$ comes from the dependence of $\tilde{u}(a)$ on $N$ in (4.9). For fixed $N>0$, the function $a \mapsto \widetilde{N}(a)$ is continuous and the same holds for the functions $a \mapsto \tilde{u}(a)$ and $a \mapsto \gamma(\tilde{u}(a))$. Moreover,

$$
\begin{gathered}
\lim _{a \rightarrow 1} \tilde{N}(a) \gamma(\tilde{u}(a)) \leq \gamma(\tilde{u}(2)) \lim _{a \rightarrow 1} \tilde{N}(a)=0, \\
\lim _{a \rightarrow+\infty} \tilde{N}(a) \gamma(\tilde{u}(a)) \geq \gamma(\tilde{u}(2)) \lim _{a \rightarrow+\infty} \tilde{N}(a)=+\infty .
\end{gathered}
$$

Therefore, for any $N>0$ the equation (4.10) has a solution $a \in(0, \infty)$. This provides a solution $(1 / a, u(1 / a))$ for $(4.9)$ and thus a SFD distribution $\tilde{\chi}$ such that $N(\widetilde{\chi})=N$ and $P(\widetilde{\chi})=P$.

In order to show that the map $N \mapsto E\left(\widetilde{\chi}_{N, P}\right)$ is increasing, we argue as follows. First, we notice that the map $N \mapsto a(N)$ with $a(N)$ such that $N=\Xi(a(N), N)$ is continuous and thus the map $E\left(\widetilde{\chi}_{., P}\right)$ is also continuous. Moreover, this map is injective. Indeed, if $N, N^{\prime}>0$ are such that $E\left(\widetilde{\chi}_{N, P}\right)=E\left(\widetilde{\chi}_{N^{\prime}, P}\right)=: E$, then using the uniqueness proved in part 1) (or also Lemma 4.3 below), we obtain $\widetilde{\chi}_{N, P}=\widetilde{\chi}_{N^{\prime}, P}=\chi_{P, E}$, and then $N=N^{\prime}$. This implies that $E\left(\tilde{\chi}_{., P}\right)$ is a monotonous function of $\mathbb{R}_{+}$. Since

$$
E\left(\widetilde{\chi}_{N, P}\right) \geq m c N \longrightarrow \infty \text { when } N \longrightarrow \infty,
$$


it follows that it may only be increasing.

LEMmA 4.3. - For any SFD state $\chi=\mathbf{1}_{\mathcal{E}}, \mathcal{E}=\left\{\beta^{0} p^{0}-\beta \cdot p \leq 1\right\}$ and any Borel set $B$, we have

$$
N\left(\mathbf{1}_{B}\right)=N(\chi), P\left(\mathbf{1}_{B}\right)=P(\chi) \quad \text { implies } \quad E\left(\mathbf{1}_{B}\right) \geq E(\chi)
$$

where the inequality is strict if $B \neq \mathcal{E}$ a.e., and

$$
P\left(\mathbf{1}_{B}\right)=P(\chi), E\left(\mathbf{1}_{B}\right)=E(\chi) \quad \text { implies } \quad N\left(\mathbf{1}_{B}\right) \leq N(\chi),
$$

and the inequality is strict if $B \neq \mathcal{E}$ a.e.

REMARK 4.4. - As a consequence of Lemma 4.3, we recover that for any given $N>0$ and $P \in \mathbb{R}^{3}$ there exists at most one SFD state $\chi$ such that $N(\chi)=N$ and $P(\chi)=P$ and that for any given $P \in \mathbb{R}^{3}$ and $E>0$ there exists at most one SFD state $\chi$ such that $P(\chi)=P$ and $E(\chi)=E$. Indeed, for example for the first claim, if $\chi=\mathbf{1}_{\mathcal{E}}$ and $\chi^{\prime}=\mathbf{1}_{\mathcal{E}^{\prime}}$ are two SFD distributions such that $N(\chi)=N\left(\chi^{\prime}\right)$ and $P(\chi)=P\left(\chi^{\prime}\right)$, we have, using twice Lemma 4.3, $E(\chi)=E\left(\chi^{\prime}\right)$ and therefore $\mathcal{E}=\mathcal{E}^{\prime}$ a.e., so that $\chi=\chi^{\prime}$.

Proof of Lemma 4.3. - We only prove (4.11) since the proof of (4.12) is exactly the same. Given a SFD $\chi=\mathbf{1}_{\mathcal{E}}$ and a Borel set $B$ satisfying $N\left(\mathbf{1}_{B}\right)=$ $N(\chi), P\left(\mathbf{1}_{B}\right)=P(\chi)$, we just compute

$$
\begin{aligned}
E\left(\mathbf{1}_{B}\right)-E(\chi) & =\int_{B} p^{0} \mathrm{~d} p-\int_{\mathcal{E}} p^{0} \mathrm{~d} p=\int_{B \backslash \mathcal{E}} p^{0} \mathrm{~d} p-\int_{\mathcal{E} \backslash B} p^{0} \mathrm{~d} p \\
& \geq \frac{1}{\beta^{0}}\left[\int_{B \backslash \mathcal{E}}(1+\beta \cdot p) d p-\int_{\mathcal{E} \backslash B}(1+\beta \cdot p) \mathrm{d} p\right] \\
& \geq \frac{1}{\beta^{0}}\left[\int_{B}(1+\beta \cdot p) \mathrm{d} p-\int_{\mathcal{E}}(1+\beta \cdot p) \mathrm{d} p\right]=0
\end{aligned}
$$

and the inequality is strict if $B \neq \mathcal{E}$ a.e.

LEMMA 4.5. - Let consider $\varepsilon: \mathbb{R}^{2} \rightarrow \mathbb{R}$ a radial and increasing (with respect to the radius) function. For any $0 \leq g \in L^{1}\left(\mathbb{R}^{2}\right)$ define $\rho \geq 0$ and $0 \leq g^{\star}$, $g^{\star} \in L^{1}\left(\mathbb{R}^{2}\right)$ by

$$
\pi \rho^{2}=\int_{\mathbb{R}^{2}} g\left(p^{\prime}\right) \mathrm{d} p^{\prime}, \quad g^{\star}=\mathbf{1}_{\left\{\left|p^{\prime}\right| \leq \rho\right\}},
$$

and for any $0 \leq \phi \in L_{\varepsilon}^{1}\left(\mathbb{R}^{2}\right)=L^{1}\left(\mathbb{R}^{2} ;\left(1+\varepsilon\left(p^{\prime}\right)\right) \mathrm{d} p^{\prime}\right)$ also define

$$
n(\phi):=\int_{\mathbb{R}^{2}} \phi\left(p^{\prime}\right) \mathrm{d} p^{\prime}, \quad e(\phi):=\int_{\mathbb{R}^{2}} \phi\left(p^{\prime}\right) \varepsilon(p) \mathrm{d} p^{\prime} .
$$

Then, for any $g \in L_{\varepsilon}^{1}$ there holds

$$
n\left(g^{\star}\right)=n(g) \quad \text { and } \quad e\left(g^{\star}\right) \leq e(g) .
$$

Proof. - It is done is several steps. 
- Step 1). Assume that $g\left(p^{\prime}\right)=\lambda \mathbf{1}_{\left\{a \leq\left|p^{\prime}\right| \leq b\right\}}$ with $0<\lambda<1$, and define

$$
g_{\lambda_{t}}\left(p^{\prime}\right)=\lambda_{t} \mathbf{1}_{\left\{a \leq\left|p^{\prime}\right| \leq b_{t}\right\}}
$$

with $b_{t}=b-t$ and $\lambda_{t}$ defined by the condition $n\left(g_{\lambda_{t}}\right)=n(g)$ for all $t \geq 0$. This condition implies that

$$
\frac{\mathrm{d}}{\mathrm{d} t}\left[n\left(g_{\lambda_{t}}\right)\right]=\frac{\mathrm{d}}{\mathrm{d} t}\left[\lambda_{t} \int_{a}^{b_{t}} r \mathrm{~d} r\right]=\lambda_{t}^{\prime} \int_{a}^{b_{t}} r \mathrm{~d} r+\lambda_{t} b_{t} b_{t}^{\prime}=0,
$$

and thus $\lambda_{t}^{\prime} \int_{a}^{b_{t}} r \mathrm{~d} r=\lambda_{t} b_{t}$. Then, we compute

$$
\begin{aligned}
\frac{\mathrm{d}}{\mathrm{d} t}\left[e\left(g_{\lambda_{t}}\right)\right] & =\frac{\mathrm{d}}{\mathrm{d} t}\left[\lambda_{t} \int_{a}^{b_{t}} \varepsilon(r) r \mathrm{~d} r\right]=\lambda_{t}^{\prime} \int_{a}^{b_{t}} \varepsilon(r) r \mathrm{~d} r+\lambda_{t} \varepsilon\left(b_{t}\right) b_{t} b_{t}^{\prime} \\
& =\frac{\lambda_{t} b_{t}}{\int_{a}^{b_{t}} r \mathrm{~d} r} \int_{a}^{b_{t}}\left(\varepsilon(r)-\varepsilon\left(b_{t}\right)\right) r \mathrm{~d} r \leq 0,
\end{aligned}
$$

and therefore, in particular, the function $g_{1}=\mathbf{1}_{\left\{a \leq\left|p^{\prime}\right| \leq c\right\}}$, uniquely defined by $n\left(g_{1}\right)=n(g)$, satisfies $e\left(g_{1}\right) \leq e(g)$.

- Step 2). Assume now that $g\left(p^{\prime}\right)=\mathbf{1}_{\left\{a \leq\left|p^{\prime}\right| \leq b\right\}}$ and let define

$$
g^{t}\left(p^{\prime}\right)=\mathbf{1}_{\left\{a_{t} \leq\left|p^{\prime}\right| \leq b_{t}\right\}}
$$

with $a_{t}=a-t$ and $b_{t}$ defined by the condition $n\left(g^{t}\right)=n(g)$ for all $t \geq 0$. This condition implies

$$
\frac{\mathrm{d}}{\mathrm{d} t}\left[n\left(g^{t}\right)\right]=\frac{\mathrm{d}}{\mathrm{d} t}\left[\int_{a_{t}}^{b_{t}} r \mathrm{~d} r\right]=\frac{1}{2}\left(b_{t} b_{t}^{\prime}-a_{t} a_{t}^{\prime}\right)=0,
$$

so that $b_{t} b_{t}^{\prime}=-a_{t}$. Then, we compute

$$
\frac{\mathrm{d}}{\mathrm{d} t}\left[e\left(g^{t}\right)\right]=\frac{\mathrm{d}}{\mathrm{d} t} \int_{a_{t}}^{b_{t}} \varepsilon(r) r \mathrm{~d} r=-a_{t}\left(\varepsilon\left(b_{t}\right)-\varepsilon\left(a_{t}\right)\right) \leq 0,
$$

and therefore, for any $t \geq 0$, we have $n\left(g^{t}\right)=n(g), e\left(g^{t}\right) \leq e(g)$.

- Step 3). Assume now that $g$ is a step function, of the form

$$
g\left(p^{\prime}\right)=\sum_{k=1}^{K} \lambda_{k} \mathbf{1}_{\left\{a_{k} \leq\left|p^{\prime}\right| \leq b_{k}\right\}}
$$

with $0<\lambda_{k} \leq 1$ and $a_{1}<b_{1} \leq a_{2}<b_{2} \leq \cdots \leq a_{K}<b_{K}$. Using Step 1) we construct $g_{1}$ of the form

$$
g_{1}\left(p^{\prime}\right)=\sum_{k=1}^{K} \mathbf{1}_{\left\{a_{k} \leq\left|p^{\prime}\right| \leq c_{k}\right\}},
$$

with $a_{1}<c_{1} \leq a_{2}<c_{2} \leq \cdots \leq a_{K}<c_{K}$, such that $n\left(g_{1}\right)=n(g)$ and $e\left(g_{1}\right) \leq e(g)$. By induction on $k=1, \ldots, K$, using Step 2) on each function $\mathbf{1}_{\left\{a_{k} \leq\left|p^{\prime}\right| \leq c_{k}\right\}}$ we obtain that (4.15) holds for any step function $g$. 
- Step 4). For a radial function $g$ we argue by density. We consider a sequence $\left(g_{n}\right)$ of step functions of the form (4.16) and such that $\lim g_{n}=g$ in $L_{\varepsilon}^{1}\left(\mathbb{R}^{2}\right)$, in particular $\lim n\left(g_{n}\right)=n(g)$ and $\lim e\left(g_{n}\right)=e(g)$. This implies that $\lim \rho_{n}=\rho$, where $\rho$ and $\left(\rho_{n}\right)$ are defined by (4.12), and therefore that $g_{n}^{\star}=\mathbf{1}_{\left\{\left|p^{\prime}\right| \leq \rho_{n}\right\}} \rightarrow g^{\star}=\mathbf{1}_{\left\{\left|p^{\prime}\right| \leq \rho\right\}}$ in $L_{\varepsilon}^{1}\left(\mathbb{R}^{2}\right)$. By Step 3), we already know that (4.13) holds for the sequence $\left(g_{n}\right)$ and we can pass to the limit: (4.13) is proved for any radial function.

- Step 5) Finally, for a general function $g$ we define the radial function

$$
\tilde{g}\left(p^{\prime}\right):=\frac{1}{2 \pi} \int_{S^{1}} g\left(\left|p^{\prime}\right| \omega\right) \mathrm{d} \omega .
$$

Since $\varepsilon$ is radial we get $n(\tilde{g})=n(g)$ and $e(\tilde{g})=e(g)$. We then apply Step 4) to conclude.

Proposition 4.6. - For any SFD state $\chi$ and any $f$ such that $0 \leq f \leq 1$, we have

$$
N(f)=N(\chi), P(f)=P(\chi) \quad \text { implies } \quad E(f) \geq E(\chi) .
$$

Proof. - Let introduce $e_{1} \in S^{2}$ such that $P=|P| e_{1}$ and let us write $p=$ $\left(p_{1}, p^{\prime}\right):=p_{1} e_{1}+p^{\prime}$ with $p^{\prime} \perp e_{1}$. We have

$$
\begin{gathered}
N(f)=\int_{\mathbb{R}} n\left(f\left(p_{1}, .\right)\right) \mathrm{d} p_{1}, \quad P(f)=\int_{\mathbb{R}} p_{1} n\left(f\left(p_{1}, .\right)\right) \mathrm{d} p_{1}, \\
E(f)=\int_{\mathbb{R}} e\left(f\left(p_{1}, .\right), p_{1}\right) \mathrm{d} p_{1},
\end{gathered}
$$

where for any $\phi \geq 0$ measurable, $n(\phi)$ is defined by (4.12) and

$$
e\left(\phi, p_{1}\right)=\int_{\mathbb{R}^{2}} \phi\left(p^{\prime}\right) \sqrt{m^{2} c^{2}+\left|p_{1}\right|^{2}+\left|p^{\prime}\right|^{2}} \mathrm{~d} p^{\prime} .
$$

For a.e. $p_{1} \in \mathbb{R}$ we define $\rho\left(p_{1}\right)$ by

$$
\pi \rho\left(p_{1}\right)^{2}=\int_{\mathbb{R}^{2}} f\left(p_{1}, p^{\prime}\right) \mathrm{d} p^{\prime}, \quad f^{\star}(p)=\mathbf{1}_{B\left(0, \rho\left(p_{1}\right)\right)}\left(p^{\prime}\right)=\mathbf{1}_{B},
$$

where $B$ is the set $B:=\left\{p \in \mathbb{R}^{3}, p_{1} \in \mathbb{R}, p^{\prime} \in B\left(0, \rho\left(p_{1}\right)\right)\right\}$. Of course, $N\left(f^{\star}\right)=N(f)$ and $P\left(f^{\star}\right)=P(f)$. Therefore thanks to Lemma 4.5, we obtain that $\mathbf{1}_{B}$ satisfies

$$
N\left(\mathbf{1}_{B}\right)=N(f), \quad P\left(\mathbf{1}_{B}\right)=P(f), \quad E\left(\mathbf{1}_{B}\right) \leq E(f),
$$

and we conclude thanks to Lemma 4.3.

Proof of part 1) of Theorem 4.1. - From Proposition 4.2 we already know that for any $P \in \mathbb{R}^{3}$ and $E>|P|$ there exists a unique SFD state $\chi_{P, E}$ such that $P\left(\chi_{P, E}\right)=P$ and $E\left(\chi_{P, E}\right)=E$. Consider now $f$ such that $0 \leq f \leq 1$ and define

$$
N:=N(f), \quad P:=P(f), \quad E:=E(f) .
$$

BULlETIN DE LA SOCIÉTÉ MATHÉMATIQUE DE FRANCE 
We have just to prove that

$$
N(f) \leq N\left(\chi_{P, E}\right)
$$

Thanks to Proposition 4.2 we know that there exists a unique SFD state $\chi_{P, E}$ such that $P\left(\chi_{P, E}\right)=P, E\left(\chi_{P, E}\right)=E$, and there also exists a unique SDF state $\tilde{\chi}_{N, P}$ such that $N\left(\widetilde{\chi}_{N, P}\right)=N$ and $P\left(\widetilde{\chi}_{N, P}\right)=P$. Moreover, from Proposition 4.6

$$
E\left(\chi_{P, E}\right)=E(f) \geq E\left(\widetilde{\chi}_{N, P}\right) .
$$

But since from Proposition 4.2 the map $N \mapsto E\left(\widetilde{\chi}_{N, P}\right)$ is increasing this implies that

$$
N\left(\chi_{P, E}\right) \geq N\left(\widetilde{\chi}_{N, P}\right)=N(f),
$$

and this conclude the proof.

Proof of part 2) of Theorem 4.1. - We only need to prove that for any $P \in \mathbb{R}^{3}$ and any $E>0$ such that $|P|<E$ and for any $N \in] 0, N\left(\chi_{P, E}\right)[$ there exists $\mathcal{F}$ a Fermi-Dirac distribution such that

$$
N(\mathcal{F})=N, \quad P(\mathcal{F})=P, \quad E(\mathcal{F})=E .
$$

It is straightforward to check that $\mathcal{F}$ realizes the moments equations (4.18) if, and only if

$$
N=N(\bar{\beta}, \mu) \gamma(u), \quad E-\frac{P}{u}=-H(\bar{\beta}, \mu), \quad E-P u=E(\bar{\beta}, \mu),
$$

where

$$
L(\bar{\beta}, \mu)=L(\overline{\mathcal{F}}), \quad \overline{\mathcal{F}}=\mathcal{F}_{\bar{\beta}, 0, \mu}=\frac{1}{\mathrm{e}^{\bar{\beta} p^{0}+\mu}+1} .
$$

We then define,

$$
u=u(\bar{\beta}, \mu):=\frac{P}{E+H(\bar{\beta}, \mu)}
$$

and reduce the system (4.19) to

$$
\left\{\begin{array}{l}
\Sigma_{E}(\bar{\beta}, \mu):=E(\bar{\beta}, \mu)+\frac{P^{2}}{E+H(\bar{\beta}, \mu)}=E, \\
\Sigma_{N}(\bar{\beta}, \mu):=N(\bar{\beta}, \mu) \gamma(u(\bar{\beta}, \mu))=N .
\end{array}\right.
$$

We conclude thanks to the following result.

Lemma 2.13. - 1) For any fixed $\mu$ the function $\Sigma_{E}(., \mu): \mathbb{R}_{+}^{\star} \rightarrow \mathbb{R}$ is continuous, decreasing,

$$
\lim _{\bar{\beta} \rightarrow 0} \Sigma_{E}(\bar{\beta}, \mu)=+\infty \quad \text { and } \quad \lim _{\bar{\beta} \rightarrow+\infty} \Sigma_{E}(\bar{\beta}, \mu)=0 .
$$

Therefore, there exists a unique $\bar{\beta}=\bar{\beta}(\mu)>0$ such that $\Sigma_{E}(\bar{\beta}, \mu)=E$ for any $\mu$. 
2) The function $\bar{\beta}: \mathbb{R} \rightarrow \mathbb{R}$ is continuous and decreasing,

$$
\lim _{\mu \rightarrow-\infty} \bar{\beta}(\mu)=+\infty=\lim _{\mu \rightarrow-\infty} \bar{\beta}(\mu)+\mu=+\infty \quad \text { and } \quad \lim _{\mu \rightarrow+\infty} \bar{\beta}(\mu)=0 .
$$

3) The function $\bar{\Sigma}: \mathbb{R} \rightarrow \mathbb{R}, \bar{\Sigma}(\mu):=\Sigma_{N}(\bar{\beta}(\mu), \mu)$ is continuous, decreasing,

$$
\lim _{\mu \rightarrow-\infty} N(\mathcal{F})=N\left(\chi_{P, E}\right) \quad \text { and } \quad \lim _{\mu \rightarrow+\infty} \bar{\Sigma}(\mu)=0 .
$$

Proof of Lemma 2.13. - The proofs of the two first points are very similar to those of the same points in Lemma 2.3 and Lemma 3.3. We therefore do not repeat them. The only new point is the third, and more precisely the behavior of $\Sigma_{N}$ for $\mu \rightarrow-\infty$. In order to prove it, let $\left(\mu_{n}\right)$ be a decreasing sequence such that $\mu_{n} \rightarrow-\infty$ and set $\bar{\beta}_{n}=\bar{\beta}\left(\mu_{n}\right)$.

- Step 1). We write

$$
0<E-P \leq E(\bar{\beta}, \mu)=\int_{\mathbb{R}^{3}} \frac{p^{0}}{\mathrm{e}^{\bar{\beta}\left(p^{0}-1\right)+\bar{\beta}+\mu}+1} \mathrm{~d} p .
$$

When $\mu \rightarrow-\infty$ we have $\bar{\beta}=\bar{\beta}(\mu) \rightarrow+\infty$, and this implies that $\bar{\beta}+\mu \rightarrow-\infty$. In particular, we have $\bar{\beta}+\mu \leq 0$ so that

$$
-\frac{\mu}{\bar{\beta}(\mu)} \geq 1
$$

- Step 2). Since

$\int_{\mathbb{R}^{3}} \frac{|z|}{\mathrm{e}^{|\bar{\beta} m c+\mu|(|z|-1)}+1} \mathrm{~d} z \geq \int_{B_{1}} \frac{|z|}{\mathrm{e}^{|\bar{\beta} m c+\mu|(|z|-1)}+1} \mathrm{~d} z \geq \int_{B_{1}} \frac{|z|}{2} \mathrm{~d} z=: C_{E}>0$ we deduce

$$
\begin{aligned}
E \geq E(\bar{\beta}, \mu) & \geq \int_{\mathbb{R}^{3}} \frac{|p|}{\mathrm{e}^{\bar{\beta}(|p|+m c)+\mu}+1} \mathrm{~d} p \\
& =\left(\frac{|\bar{\beta} m c+\mu|}{\bar{\beta}}\right)^{4} \int_{\mathbb{R}^{3}} \frac{|z|}{\mathrm{e}^{|\bar{\beta} m c+\mu|(|z|-1)}+1} \mathrm{~d} z \geq\left(\frac{|\bar{\beta} m c+\mu|}{\bar{\beta}}\right)^{4} C_{E} .
\end{aligned}
$$

Therefore

$$
\left|\frac{\mu_{n}}{\bar{\beta}_{n}}\right| \leq m c+\left|m c+\frac{\mu_{n}}{\bar{\beta}_{n}}\right| \leq\left(\frac{E}{C_{E}}\right)^{1 / 4}+m c,
$$

and there exists $a \in \mathbb{R}$ and a subsequence $\left(n^{\prime}\right)$ such that $\lim \mu_{n^{\prime}} / \bar{\beta}_{n^{\prime}}=-a$, and by (4.22) $a \geq 1$.

- Step 3). We remark now that if $n$ is large enough $\beta_{n} \geq 1$ and $\left|\mu_{n}\right| / \beta_{n} \leq 2 a$, from where we deduce that

$$
0 \leq \frac{1}{\mathrm{e}^{\beta_{n}\left(p^{0}-\left|\mu_{n}\right| / \beta_{n}\right)}+1} \leq \mathbf{1}_{p^{0} \leq 2 a}+\frac{1}{\mathrm{e}^{p^{0}-2 a}+1} \mathbf{1}_{p^{0} \geq 2 a} \in L^{1}\left(\mathbb{R}^{3}\right) .
$$

Moreover, for a.e. $p \in \mathbb{R}^{3}$ we have

$$
\mathcal{F}_{n}=\frac{1}{\mathrm{e}^{\beta_{n}\left(p^{0}-\left|\mu_{n}\right| / \beta_{n}\right)}+1} \longrightarrow \mathbf{1}_{\left\{p^{0} \leq a\right\}} .
$$

BULlETIN DE LA SOCIÉTÉ MATHÉMATIQUE DE FRANCE 
We conclude by dominated convergence Lebesgue Theorem that

$$
L\left(\bar{\beta}_{n^{\prime}}, \mu_{n^{\prime}}\right)=L\left(\overline{\mathcal{F}}_{n^{\prime}}\right) \rightarrow \widetilde{L}(a):=L\left(\mathbf{1}_{\left\{p^{0} \leq a\right\}}\right) \quad \text { for } \quad L=N, E, G, H .
$$

- Step 4). Passing to the limit in (4.20) and (4.21) we obtain

$$
E=\widetilde{E}(a)+P \frac{\tilde{u}(a)}{c}, \quad \tilde{u}(a)=\frac{P}{E+\widetilde{H}(a)}
$$

and $\lim \bar{\Sigma}_{N}(\mu)=\widetilde{N}(a) \gamma(\tilde{u}(a))$ when $\mu \rightarrow-\infty$. By (4.6) this means precisely that the SFD state associated to $(\bar{\beta}, u)=(1 / a, \tilde{u}(1 / a))$ satisfies

$$
\lim _{\mu \rightarrow-\infty} \bar{\Sigma}_{N}(\mu)=N(\chi), \quad P(\chi)=P, \quad E(\chi)=E .
$$

Therefore, $\chi=\chi_{P, E}$ and $\bar{\Sigma}_{N}(\mu)=N(\mathcal{F}) \rightarrow N\left(\chi_{P, E}\right)$.

Nonrelativistic Fermi-Dirac particles. - Here again, since the energy is $\mathcal{E}(p)=\left|p^{2}\right| / 2 m$ the problem (1.2) is equivalent to (3.16): given three quantities $N>0, E>0, P \in \mathbb{R}^{3}$ find $f(p)$ such that $0 \leq f \leq 1$ and satisfying (3.16).

On the other hand, given $N>0$ and $P \in \mathbb{R}^{3}$, we have

$$
\begin{gathered}
\min _{\substack{0 \leq f \leq 1 \\
\int f \mathrm{~d} p=N \\
\int p f(p) \mathrm{d} p=P}} \frac{1}{2 m} \int_{\mathbb{R}^{3}}|p|^{2} f(p) \mathrm{d} p \\
=\min _{\substack{0 \leq g \leq 1 \\
\int g \mathrm{~d} p=N\\
}} \frac{1}{2 m} \int_{\mathbb{R}^{3}}\left|p-\frac{P}{N}\right|^{2} g(p) \mathrm{d} p, \\
\qquad(p-P / N) g(p) \mathrm{d} p=0
\end{gathered}
$$

and this minimum is reached for a distribution of the form $g(p)=\mathbf{1}_{\{|p-P / N| \leq c\}}$, for some $c>0$ and for a distribution of the form $f(p)=\mathbf{1}_{\{|p| \leq c\}}$. Since

$$
\int_{\mathbb{R}^{3}} \mathbf{1}_{\{|p| \leq c\}} \mathrm{d} p=\omega_{2} \frac{c^{3}}{3} \text { and } \int_{\mathbb{R}^{3}}\left|p^{2}\right| \mathbf{1}_{\{|p| \leq c\}} \mathrm{d} p=\omega_{2} \frac{c^{5}}{5}
$$

we deduce that the problem (1.2) has a solution $f$ satisfying $0 \leq f \leq 1$ only if

$$
E \geq \frac{3^{5 / 3}(4 \pi)^{2 / 3}}{5} N^{5 / 3} .
$$

On the other hand, it is simple, using elementary calculus, to prove that for any $E, N>0, P \in \mathbb{R}^{3}$ such that (4.21) holds, there exists a distribution of the from

$$
F(p)=\left\{\begin{array}{lll}
\frac{1}{\mathrm{e}^{a|p-P / N|^{2}+b}+1} & \text { if } & E>\frac{3^{5 / 3}(4 \pi)^{2 / 3}}{5} N^{5 / 3}, \\
\mathbf{1}_{\{|p-P / N| \leq c\}} & \text { if } & E=\frac{3^{5 / 3}(4 \pi)^{2 / 3}}{5} N^{5 / 3}
\end{array}\right.
$$

with $a \in \mathbb{R}, b \in \mathbb{R}$ and $c \in \mathbb{R}$ such that $\omega_{2} c^{3} / 3=N$ satisfying (3.16). 
Then, for every $E, N>0$ and $P \in \mathbb{R}^{3}$ satisfying (4.23), the Fermi Dirac distribution

$$
\mathcal{F}(p)= \begin{cases}\frac{1}{\mathrm{e}^{\nu(p)}+1}, \nu(p)=a|p|^{2}-\frac{2 a}{N} P \cdot p+ & \left(b+\frac{a|P|^{2}}{N^{2}}\right) \\ & \text { if } \quad E>\frac{3^{5 / 3}(4 \pi)^{2 / 3}}{5} N^{5 / 3} \\ \mathbf{1}_{\{|p-P / N| \leq c\}} & \text { if } \quad E=\frac{3^{5 / 3}(4 \pi)^{2 / 3}}{5} N^{5 / 3}\end{cases}
$$

solves (1.2).

This shows that for non relativistic particles Theorem 4.1 remains valid under the unique following change: points 1) and 2) have to be replaced by

$\left.1^{\prime}\right)$ For every $E, N>0, P \in \mathbb{R}^{3}$, satisfying (4.23) there exists a non relativistic fermi Dirac state, saturated or not, defined by (4.25) corresponding to these moments, i.e. satisfying (1.2).

Points 3) and 4) of Theorem 4.1 remain unchanged.

\section{Appendix. Minkowsky space and Lorentz transform}

For the convenience of the reader, we sumarize some of well-known facts on Minkowsky space and Lorentz transform that we have systematically used in the construction of the equilibrium states with prescribed moments. We refer to $[20]$ for a more detailed exposition on the subject.

Let us denote $P=\left(P^{0}, p\right) \in \mathbb{R}^{4}$ with $P^{0} \in \mathbb{R}, p \in \mathbb{R}^{3}$ or indifferently $P=\left(P^{\mu}\right)$. We define the Lorentz metric as follows:

$$
\langle P, Q\rangle=P^{0} Q^{0}-p \cdot q, \quad \forall P, Q \in \mathbb{R}^{4} .
$$

We also write

$$
\langle P, Q\rangle=P^{\mu} Q_{\mu}=P^{\top} \eta Q=\sum_{\mu, \nu=0}^{4} \eta_{\mu \nu} P^{\mu} Q^{\nu},
$$

with $Q_{\mu}=\eta_{\mu \nu} Q^{\mu}$, where

$$
\eta=\left(\eta_{\mu \nu}\right)=\left(\begin{array}{cc}
1 & 0^{\top} \\
0 & -\mathbf{I}_{3}
\end{array}\right)
$$

is the Minkowsky matrix. The inner product $\langle$,$\rangle on \mathbb{R}^{4}$ is symmetric, non degenerated but not positive.

Definition A.1. - A Lorentz transform is a linear operator $\Lambda: \mathbb{R}^{4} \rightarrow \mathbb{R}^{4}$ such that

$$
\langle\Lambda P, \Lambda Q\rangle=\langle P, Q\rangle, \quad \forall P, Q \in \mathbb{R}^{4} .
$$

BULLETIN DE LA SOCIÉTÉ MATHÉMATIQUE DE FRANCE 
Example 1: Rotations. - For any rotation $\mathbf{R}$ of $\mathbb{R}^{3}(\mathbf{R} \in \mathrm{SO}(3))$,

$$
\Lambda=\left(\begin{array}{ll}
1 & 0 \\
0 & \mathbf{R}
\end{array}\right)
$$

is a Lorentz transform.

Example 2: Boosts. - For any $v \in \mathbb{R}^{3}$ such that $v:=|v|<1$,

$$
\Lambda=\left(\begin{array}{cc}
\gamma & \gamma v^{\top} \\
\gamma v & \mathbf{I}+(\gamma-1) / v^{2} \cdot v v^{\top}
\end{array}\right), \quad \gamma=\frac{1}{\sqrt{1-v^{2}}}
$$

is a Lorentz transform.

REMARK A.2. - Any Lorentz transform is the composition of a boost and a rotation.

For $\left(\beta^{0}, \beta\right) \in \mathbb{R}^{4}$ with $\beta^{0}>|\beta|$ we define $\bar{\beta}>0$ and $u \in \mathbb{R}^{3}$ by

$$
\bar{\beta}^{2}:=\left(\beta^{0}\right)^{2}-|\beta|^{2}, \quad \frac{u}{c}:=\frac{\beta}{\beta^{0}} .
$$

Then, setting $\gamma:=\left(1-(u / c)^{2}\right)^{-1 / 2}$ we have

$$
\left(\beta^{\mu}\right)=\left(\beta^{0}, \beta\right)=\left(\gamma \bar{\beta}, \gamma \bar{\beta} \frac{u}{c}\right) .
$$

For such a 4 -vector $\left(\beta^{0}, \beta\right)$ we define the boost transform $\Lambda=\Lambda_{\left(\beta^{\mu}\right)}$ associated to $v:=u / c$. It satisfies

$$
\Lambda\left(\begin{array}{c}
\bar{\beta} \\
0
\end{array}\right)=\left(\begin{array}{c}
\beta^{0} \\
\beta
\end{array}\right) .
$$

Lemma A.3. - Any Lorentz transform $\Lambda$ satisfies $\operatorname{det} \Lambda= \pm 1$.

Proof. - Define

$$
\Lambda^{\star}=\left(\begin{array}{cc}
a & -c^{\top} \\
-b & d^{\top}
\end{array}\right) \quad \text { for } \quad \Lambda=\left(\begin{array}{ll}
a & b^{\top} \\
c & d
\end{array}\right)
$$

with $a \in \mathbb{R}, b, c \in \mathbb{R}^{3}$ and $d \in M\left(\mathbb{R}^{3}\right)$. We easily verify that

$$
\langle\Lambda P, Q\rangle=\left\langle P, \Lambda^{\star} Q\right\rangle \quad \text { for any } P, Q \in \mathbb{R}^{4} .
$$

In particular, by definition of a Lorentz transform, one has

$$
\left\langle\Lambda^{\star} \Lambda P, Q\right\rangle=\langle\Lambda P, \Lambda Q\rangle=\langle P, Q\rangle \quad \text { for any } P, Q \in \mathbb{R}^{4},
$$

so that $\Lambda^{\star} \Lambda=\operatorname{Id}_{4}$. Since $\operatorname{det} \Lambda^{\star}=\operatorname{det} \Lambda$, we $\operatorname{get}(\operatorname{det} \Lambda)^{2}=1$.

Definition A.4. - For $s \in \mathbb{R}$ we define the hyperboloid

$$
M_{+}^{s}:=\left\{P \in \mathbb{R}^{4} ;\langle P, P\rangle=s, P^{0}>0\right\}=\left\{\left(\sqrt{s+|p|^{2}}, p\right) ; p \in \mathbb{R}^{3}\right\} .
$$

We write $\Lambda \in \mathcal{L}_{+}^{\uparrow}$, if $\Lambda$ is a Lorentz transform such that $\operatorname{det} \Lambda=+1$ and $(\Lambda P)^{0}>0$ for any $P \in M_{+}^{s}$, with $s>0$. 
The boost $\Lambda$ associated to $\left(\beta^{0}, \beta\right)$ belongs to $\mathcal{L}_{+}^{\uparrow}$ since $\operatorname{det} \Lambda=1$ and

$$
(\Lambda P)^{0}=\gamma p^{0}+\gamma u \cdot p=\frac{1}{\bar{\beta}}\left(\beta^{0} p^{0}+\beta \cdot p\right) \geq \frac{1}{\bar{\beta}}\left(\beta^{0}|p|-|\beta| \cdot|p|\right) \geq 0
$$

for any $P \in M_{+}^{s}$ with $s>0$.

Lemma A.5. - Let $f: \mathbb{R} \rightarrow \mathbb{R}$ and $\left(\beta^{\mu}\right)=\left(\beta^{0}, \beta\right) \in \mathbb{R}^{4}$ such that $\bar{\beta}^{2}:=$ $\beta^{02}-|\beta|^{2}>0$. We define $F: \mathbb{R}^{3} \rightarrow \mathbb{R}$ by

$$
F(p)=f\left(\beta^{\mu} p^{\mu}\right)=f\left(\beta^{0} p^{0}-\beta \cdot p\right)
$$

with $p^{0}:=\sqrt{s+|p|^{2}}, s>0$. There is some constants $A_{i}=A_{i}(f, \bar{\beta})$ such that

$$
\begin{gathered}
\int_{\mathbb{R}^{3}} p^{\mu} F \frac{\mathrm{d} p}{p^{0}}=A_{1} \beta^{\mu} \\
\int_{\mathbb{R}^{3}} p^{\mu} p^{\nu} F \frac{\mathrm{d} p}{p^{0}}=A_{2} \eta^{\mu \nu}+A_{3} \beta^{\mu} \beta^{\nu} .
\end{gathered}
$$

In particular, one has

$$
\begin{gathered}
N(F):=\int_{\mathbb{R}^{3}} F \mathrm{~d} p=A_{1} \beta^{0}, \\
P(F):=\int_{\mathbb{R}^{3}} F p \mathrm{~d} p=A_{3} \beta^{0} \beta, \\
E(F):=\int_{\mathbb{R}^{3}} F p^{0} \mathrm{~d} p=A_{2}+A_{3}\left(\beta^{0}\right)^{2}, \\
G(F):=s \int_{\mathbb{R}^{3}} F \frac{\mathrm{d} p}{p^{0}}=4 A_{2}+A_{3} \bar{\beta}^{2} .
\end{gathered}
$$

Before proving this result, let us recall and prove the following elementary distributionnal lemma.

Lemma A.6. - For any $a, b \in \mathbb{R}, a \neq b$, one has

$$
\delta_{(x-a)(x-b)}=\frac{1}{|b-a|}\left(\delta_{x-a}+\delta_{x-a}\right) .
$$

Proof. - Let $\left(\rho_{\varepsilon}\right)$ be a sequence of $L^{1}(\mathbb{R})$ such that $\rho_{\varepsilon} \rightarrow \delta$ in $\mathcal{D}^{\prime}(\mathbb{R})$. Then

$$
\begin{aligned}
\left\langle\delta_{(x-a)(x-b)}, \phi\right\rangle & :=\lim _{\varepsilon \rightarrow 0}\left\langle\rho_{\varepsilon}((x-a)(x-b)), \phi\right\rangle \\
& =\lim _{\varepsilon \rightarrow 0} \int_{\mathbb{R}} \rho_{\varepsilon}((x-a)(x-b)) \phi \mathrm{d} x=\lim _{\varepsilon \rightarrow 0}\left(I_{\varepsilon}+J_{\varepsilon}\right),
\end{aligned}
$$

with

$I_{\varepsilon}=\int_{-\infty}^{(a+b) / 2} \rho_{\varepsilon}((x-a)(x-b))=\phi \mathrm{d} x, \quad J_{\varepsilon}=\int_{(a+b) / 2}^{\infty} \rho_{\varepsilon}((x-a)(x-b)) \phi \mathrm{d} x$.

Without loss of generality we may assume that $a<b$. Set $y=(x-a)(x-b)=$ $x^{2}-(a+b) x+a b$. The function $x \mapsto y(x)$ is monotonous for any $x \leq \frac{1}{2}(a+b)$ so BULlETIN DE LA SOCIÉTÉ MATHÉMATIQUE DE FRANCE 
that it is an allowed change of variable. We compute $2 x=a+b \pm \sqrt{4 y+(a-b)^{2}}$ and $\mathrm{d} y=[2 x-(a+b)] \mathrm{d} x= \pm \sqrt{4 y+(a-b)^{2}} \mathrm{~d} x$, and hence

$$
\begin{aligned}
I_{\varepsilon}=\int_{-(b-a)^{2} / 4}^{\infty} \rho_{\varepsilon}(y) \phi\left(\frac{a+b}{2}\right. & \left. \pm \sqrt{y+\left(\frac{1}{2}(a-b)\right)^{2}}\right) \frac{\mathrm{d} y}{\sqrt{4 y+(a-b)^{2}}} \\
& \longrightarrow \frac{1}{|b-a|} \phi\left(\frac{a+b-|a-b|}{2}\right)=\frac{\phi(a)}{|b-a|} .
\end{aligned}
$$

Similarly, we prove $\lim J_{\varepsilon}=\phi(a) /|b-a|$.

Proof of Lemma A.5. - Using Lemma A.6 we have, denoting by $H$ the Heavside function,

$$
\delta_{P^{2}=s} H\left(P^{0}\right)=\delta_{\left(P^{0}\right)^{2}-s} \mathbf{1}_{P^{0}>0}=\frac{1}{2 \sqrt{s+|p|^{2}}} \delta_{P^{0}-\sqrt{s+|p|^{2}}} .
$$

Therefore, we get the fundamental identity

$$
\begin{aligned}
\int_{\mathbb{R}^{4}} F(P) \delta_{P^{2}=s} H\left(p^{0}\right) \mathrm{d} P & =\int_{\mathbb{R}^{3}}\left\{\int_{\mathbb{R}} F(P) \frac{1}{2 \sqrt{s+|p|^{2}}}=\delta_{P^{0}-\sqrt{s+|p|^{2}}} P^{0}\right\} \mathrm{d} p \\
& =\int_{\mathbb{R}^{3}} F\left(p^{0}, p\right) \frac{\mathrm{d} p}{p^{0}} .
\end{aligned}
$$

For $\Lambda \in \mathcal{L}_{+}^{\uparrow}$ we get

$$
\begin{aligned}
\int_{\mathbb{R}^{3}} F\left(\Lambda\left(p^{0}, p\right)\right) \frac{\mathrm{d} p}{p^{0}} & =\int_{\mathbb{R}^{4}} F(\Lambda P) \delta_{P^{2}=s} H\left(p^{0}\right) \mathrm{d} P \\
& =\int_{\mathbb{R}^{4}} F(Q) \delta_{Q^{2}=s} H\left(q^{0}\right) \mathrm{d} Q=\int_{\mathbb{R}^{3}} F\left(p^{0}, p\right) \frac{\mathrm{d} p}{p^{0}} .
\end{aligned}
$$

Now we choose as $\Lambda$ the boost associated to $\left(\beta^{\mu}\right)$ and using (A.8) we have, setting $P=\Lambda Q$, we get

$$
\begin{aligned}
\int_{\mathbb{R}^{3}} p^{\mu} f\left(\beta^{\nu} p^{\nu}\right) \frac{\mathrm{d} p}{p^{0}} & =\int_{\mathbb{R}^{3}} p^{\mu} f\left(\bar{\beta}\left(\Lambda^{-1} P\right)^{0}\right) \frac{\mathrm{d} p}{p^{0}} \\
& =\Lambda^{\mu} \int_{\mathbb{R}^{3}} q f\left(\bar{\beta} q^{0}\right) \frac{\mathrm{d} q}{q^{0}}=\Lambda^{\mu}\left(\begin{array}{c}
\bar{\beta} A_{1} \\
0
\end{array}\right),
\end{aligned}
$$

with $A_{1}:=\frac{1}{\bar{\beta}} \int_{\mathbb{R}^{3}} q^{0} f\left(\bar{\beta} q^{0}\right) \frac{\mathrm{d} q}{q^{0}}$, since

$$
\int_{\mathbb{R}^{3}} q^{i} f\left(\bar{\beta} q^{0}\right) \frac{\mathrm{d} q}{q^{0}}=0
$$

for $i=1,2,3$ by rotation symmetry. This proves (A.10). Similarly,

$$
\int_{\mathbb{R}^{3}} p^{\mu} p^{\nu} f\left(\beta^{\sigma} p^{\sigma}\right) \frac{\mathrm{d} p}{p^{0}}=\Lambda_{\mu^{\prime}}^{\mu} \Lambda_{\nu^{\prime}}^{\nu} \int_{\mathbb{R}^{3}} q^{\mu^{\prime}} q^{\nu^{\prime}} f\left(\bar{\beta} q^{0}\right) \frac{\mathrm{d} q}{q^{0}}=\Lambda_{\mu^{\prime}}^{\mu} \Lambda_{\nu^{\prime}}^{\nu} \alpha_{\mu^{\prime}} \delta_{\mu^{\prime} \nu^{\prime}}
$$

TOME $133-2005-\mathrm{N}^{\mathrm{O}} 1$ 
with

$$
\alpha_{0}=\int_{\mathbb{R}^{3}}\left(q^{0}\right)^{2} f\left(\bar{\beta} q^{0}\right) \frac{\mathrm{d} q}{q^{0}} \quad \alpha_{1}=\alpha_{2}=\alpha_{3}=\int_{\mathbb{R}^{3}}\left(q^{1}\right)^{2} f\left(\bar{\beta} q^{0}\right) \frac{\mathrm{d} q}{q^{0}} .
$$

Just compute

$$
\begin{aligned}
\Lambda^{2} & =\left(\begin{array}{cc}
2 \gamma^{2}-1 & 2 \gamma^{2} v^{\top} \\
2 \gamma^{2} v & \mathbf{I}+2 \gamma^{2} v v^{\top}
\end{array}\right) \\
& =-(\eta)^{\mu \nu}+2\left(\begin{array}{cc}
\gamma^{2} & \gamma(\gamma u / c)^{\top} \\
\gamma(\gamma u / c) & (\gamma u / c)(\gamma u / c)^{\top}
\end{array}\right)=-(\eta)^{\mu \nu}+\frac{2}{\bar{\beta}^{2}} \beta^{\mu} \beta^{\nu}, \\
\Lambda_{0}^{\mu} & =\left(\begin{array}{c}
\gamma \\
\gamma u / c
\end{array}\right)=\frac{\beta^{\mu}}{\bar{\beta}} .
\end{aligned}
$$

Therefore

$$
\begin{aligned}
\int_{\mathbb{R}^{3}} p^{\mu} p^{\nu} f\left(\beta^{\sigma} p^{\sigma}\right) \frac{\mathrm{d} p}{p^{0}} & =\alpha_{1} \Lambda_{\mu^{\prime}}^{\mu} \Lambda_{\mu^{\prime}}^{\nu}+\left(\alpha_{0}-\alpha_{1}\right) \Lambda_{0}^{\mu} \Lambda_{0}^{\nu} \\
& =\alpha_{1}\left(\Lambda^{2}\right)^{\mu \nu}+\left(\alpha_{0}-\alpha_{1}\right) \Lambda_{0}^{\mu} \Lambda_{0}^{\nu}=-\alpha_{1} \eta^{\mu \nu}+\frac{\alpha_{0}+\alpha_{1}}{\bar{\beta}^{2}} \beta^{\mu} \beta^{\nu}
\end{aligned}
$$

and (A.11) is proved. The points (A.12), (A.13) and (A.14) follow. Finally, we compute, thanks to (A.11),

$$
\int_{\mathbb{R}^{3}} \eta_{\mu \nu} p^{\mu} p^{\nu} f \frac{\mathrm{d} p}{p^{0}}=A_{1} \eta^{\mu \nu} \eta_{\mu \nu}+A_{2} \beta^{\mu} \beta^{\nu} \eta_{\mu \nu}
$$

so that

$$
\int_{\mathbb{R}^{3}}\left(\left(p^{0}\right)^{2}-|p|^{2}\right) f \frac{\mathrm{d} p}{p^{0}}=4 A_{1}+A_{2}\left[\left(\beta^{0}\right)^{2}-|\beta|^{2}\right]=4 A_{1}+A_{2} \bar{\beta}^{2},
$$

and (A.15) follows, remarking that $\left(p^{0}\right)^{2}-|p|^{2}=s$.

\section{BIBLIOGRAPHY}

[1] AndréAsson (H.) - Regularity of the gain term and strong $L^{1}$ convergence to equilibrium for the relativistic Boltzmann equation, SIAM J. Math. Anal., t. 27 (1996), no. 5, pp. 1386-1405.

[2] Bose (S.N.) - Plancks Gesetz und Lichtquantenhypothese, Z. Phys., t. 26 (1924), pp. 178-181.

[3] Caflisch (R.E.) \& Levermore (C.D.) - Equilibrium for radiation in a homogeneous plasma, Phys. Fluids, t. 29 (1986), pp. 748-752.

[4] Cercignani (C.) - The Boltzmann equation and its applications, Applied Math. Sciences, vol. 67, Springer Verlag, 1988.

[5] Chernikov (N.A.) - Equilibrium distribution of the relativistic gas, Acta Phys. Polon., t. 26 (1964), pp. 1069-1092.

[6] Demengel (F.) \& Temam (R.) - Convex functions of a measure and applications, Indiana Univ. Math. J., t. 33 (984), no. 5, pp. 673-709.

BULlETIN DE LA SOCIÉTÉ MATHÉMATIQUE DE FRANCE 
[7] Dolbeault (J.) - Kinetic models and quantum effects, a modified Boltzmann equation for Fermi-Dirac particles, Arch. Rat. Mech. Anal., t. 127, pp. 101-131.

[8] DudyŃski (M.) \& Ekiel-JeŻEwska (M.L.) - Global existence proof for relativistic Boltzmann equation, J. Statist. Phys., t. 66 (1992), no. 2,3, pp. 991-1001.

[9] Einstein (A.) - Quantentheorie des einatomingen idealen Gases, Stiz. Presussische Akademie der Wissenschaften Phys-math. Klasse, Sitzungsberichte, t. 23 (1925), pp. 3-14.

[10] _, Zur Quantentheorie des idealen Gases, Stiz. Presussische Akademie der Wissenschaften, Phys-math. Klasse, Sitzungsberichte, t. 23 (1925), pp. 18-25.

[11] Escobedo (M.) \& Mischler (S.) - On a quantum Boltzmann equation for a gas of photons, J. Math. Pures Appl., t. 80 (2001), no. 55, pp. 471515.

[12] Escobedo (M.), Mischler (S.) \& Valle (M.A.) - Homogeneous Boltzmann equation for quantum and relativistic particles, Electronic J. Diff. Eqns. Monographs, vol. 4, 2003, http://ejde.math.swt.edu.

[13] Glassey (R.T.) - The Cauchy Problem in Kinetic Theory, SIAM, Philadelphia, 1996.

[14] Glassey (R.T.) \& Strauss (W.A.) - Asymptotic stability of the relativistic Maxwellian, Publ. Res. Inst. Math. Sciences, Kyoto University, t. 29 (1993), no. 2.

[15] — Asymptotic stability of the relativistic Maxwellian via fourteen moments, Transport Theory Stat. Physics, t. 24 (1995), pp. 657-678.

[16] Groot (S.R.), van Leeuwen (W.A.) \& van Weert (Ch.G.) - Relativistic kinetic theory, North Holland Publishing Company, 1980.

[17] LU (X.G.) - A modified Boltzmann equation for Bose-Einstein particles: isotropic solutions and long time behavior, J. Statist. Phys., t. 98 (2000), no. 5,6, pp. $1335-1394$.

[18] _ On spatially homogeneous solutions of a modified Boltzmann equation for Fermi-Dirac particles, J. Statist. Phys., t. 105 (2001), no. 1,2, pp. 353-388.

[19] Lu (X.G.) \& WennBerg (B.) - On stability and strong convergence for the spatially homogeneous Boltzmann equation for Fermi-Dirac particles, Arch. Rat. Mech. Anal., t. 168 (2003), no. 1, pp. 1-34.

[20] Naber (G.L.) - The geometry of Minkowski spacetime, Springer Verlag, 1992.

[21] Pathria (R.K.) - Statistical Mechanics, Pergamon Press, 1972.

[22] Villani (C.) - A review of mathematical topics on collisional kinetic theory, in Handbook of Mathematical Fluid Mechanics, Vol. I (Friedlander (S.) \& Serre (D.), eds.), North Holland, Amsterdam, 2002. 